\title{
Determination of the electron temperature in the modified ionosphere over HAARP using the HF pumped Stimulated Brillouin Scatter (SBS) emission lines
}

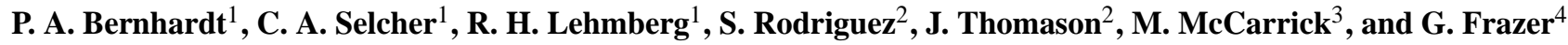 \\ ${ }^{1}$ Plasma Physics Division, Naval Research Laboratory, Washington, D.C. 20375, USA \\ ${ }^{2}$ Radar Division, Naval Research Laboratory, Washington, D.C. 20375, USA \\ ${ }^{3}$ BAE Systems, Washington, D.C., USA \\ ${ }^{4}$ ISR Division, DSTO, Edinburgh, SA, Australia
}

Received: 20 April 2009 - Revised: 23 August 2009 - Accepted: 17 September 2009 - Published: 4 December 2009

\begin{abstract}
An ordinary mode electromagnetic wave can decay into an ion acoustic wave and a scattered electromagnetic wave by a process called stimulated Brillouin scatter (SBS). The first detection of this process during ionospheric modification with high power radio waves was reported by Norin et al. (2009) using the HAARP transmitter in Alaska. Subsequent experiments have provided additional verification of this process and quantitative interpretation of the scattered wave frequency offsets to yield measurements of the electron temperatures in the heated ionosphere. Using the SBS technique, electron temperatures between 3000 and $4000 \mathrm{~K}$ were measured over the HAARP facility. The matching conditions for decay of the high frequency pump wave show that in addition to the production of an ion-acoustic wave, an electrostatic ion cyclotron wave may also be produced by the generalized SBS processes. Based on the matching condition theory, the first profiles of the scattered wave amplitude are produced using the stimulated Brillouin scatter (SBS) matching conditions. These profiles are consistent with maximum ionospheric interactions at the upper-hybrid resonance height and at a region just below the plasma resonance altitude where the pump wave electric fields reach their maximum values.
\end{abstract}

Keywords. Electromagnetics (Wave propagation) - Ionosphere (Active experiments) - Radio science (Waves in plasma)

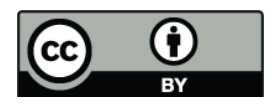

Correspondence to: P. A. Bernhardt (paul.bernhardt@nrl.navy.mil)

\section{Introduction}

Many types of waves exist in the ionospheric F-region plasma that is primarily composed of electrons and $\mathrm{O}^{+}$ ions in the earth's magnetic field. The waves supported in this plasma include O-mode and X-mode electromagnetic waves, electron plasma waves, ion acoustic waves, upper hybrid waves, lower hybrid waves, electron and ion Bernstein waves, electron whistler waves, and ion cyclotron waves. A high power electromagnetic wave from a ground transmitter can act as a pump to excite a pair of growing waves through the parametric decay instability. An overview of both observations and theory of parametric decay processes in the ionosphere pumped by high power EM waves is given by the detailed monograph on stimulated electromagnetic emissions (SEE) by Leyser (2001). Parametric decay processes that have been postulated to occur in the magnetized ionosphere include the decay of the O-mode EM wave into (a) an ion acoustic and an electron plasma wave, (b) a lower hybrid and upper hybrid wave, and (c) a lower hybrid and electron Bernstein wave.

High power lasers also interact with plasmas to produce similar decay processes. Overviews of laser plasma interactions have been provided by Kruer (1988) and by Eliezer (2002). Laser induced parametric decay processes that have been detected in an unmagnetized plasma include the decay of the light wave into (1) an ion acoustic and an electron plasma wave, (2) an ion acoustic and an electromagnetic wave, (3) an electron plasma and an electromagnetic wave, and (4) two electron plasma waves. Until recently, only the first process called the decay instability has been detected in both the high power HF wave-ionospheric and laser-plasma experiments. 
With the recent upgrade of the High Frequency Active Auroral Research Program (HAARP) facility in Alaska, it is likely that some new parametric instability will be detected in the ionospheric interactions processes. The HAARP ionospheric modification system is comprised of 180 crossed dipoles in a $12 \times 15$ array. Each dipole is fed by a separate $10 \mathrm{~kW}$ transmitter that allows separate excitation of the phase and amplitude. With all transmitters operating simultaneously, a total of 3.6 MW power can be supplied to the antenna array. The effective radiated power (ERP or the product of transmitter power and antenna gain) for the array varies with frequency. The results reported here are for operation at 4.5 and $5.6 \mathrm{MHz}$ with effective radiated powers greater than 1 gigawatt. The HAARP beam was phased for O-Mode leftcircular polarization to allow propagation of the HF wave for interactions near the critical layer where the wave frequency is equal to the local plasma frequency.

In a recent paper, Norin et al. (2009) have produced the first detection in the ionosphere of an electromagnetic pump from the ground exciting the stimulated Brillouin scatter (SBS) instability using transmissions from the HAARP facility in Alaska. They recorded the stimulated electromagnetic emissions (SEE) in a $100 \mathrm{~Hz}$ band near the pump frequency. The emissions that occurred near $\pm 9 \mathrm{~Hz}$ of the pump frequency were attributed to interactions near the HF wave reflection altitude where the wave frequency is equal to the local value of plasma frequency. Another set of emissions near $\pm 25 \mathrm{~Hz}$ were attributed to interactions near the upper hybrid resonance altitude where the pump frequency equals the local upper hybrid frequency.

Norin et al. (2009) successfully argue that the recorded scattered EM waves arise from process (2) for the laser plasma interactions where an electromagnetic wave decays into an ion acoustic wave and a scattered electromagnetic wave. In the SBS process, density fluctuations associated with ion acoustic waves couple with the pump electromagnetic wave to produce the scattered electromagnetic wave. This scattered EM wave may in turn couple with the pump wave to reinforce the ion acoustic wave. These couplings can lead to the growth of scattered electromagnetic waves along with ion acoustic waves giving the Brillouin instability (Kruer, 1988; Eliezer, 2002).

Stimulated Brillouin scattering can be inhibited by both collisional and Landau damping in the ionosphere. Several factors favor the production of SBS in the ionosphere. The electron-neutral collision frequency in the ionosphere is relatively low during the current period of solar minimum because of low atmospheric neutral densities. Second, the HAARP facility has been recently upgraded to a $12 \times 15$ antenna array with a total of 3.6 megaWatt power feeding this array. With more power, the HF beam will provide an electric field that can more often exceed the threshold for SBS and also produce larger increases in electron temperature. Higher electron temperatures are also found when heating plasmas of lower density because of reduced cooling of the elec- trons onto the ions (Djuth et al., 1987). The elevated electron temperatures during ionospheric heating greatly reduce both electron-ion collision frequencies and Landau damping of ion acoustic waves.

The primary purpose of this paper is to extend the work of Norin et al. (2009) by developing a quantitative theory of SBS in a magneto-plasma like the ionosphere. The wave number and wave frequency matching conditions are solved with the dispersion equations for the ion acoustic and electromagnetic waves keeping all of the effects of the ion and electron gyro frequencies. These solutions provide an analytic expression that will yield measurements of the electron temperatures in the upper hybrid interaction regions based on the frequencies of the widely spaced SBS lines.

This paper also presents new measurements of the SBS lines for frequencies or conditions not explored in the previous work by Norin et al. (2009). With both increased power for electromagnetic pump at HAARP and low neutral densities in the upper atmosphere, the conditions are ideal for experimental studies of SBS. On 24 October 2008, a digital receiver called the GBOX5 developed at DSTO in Australia was operated near the HAARP transmitter to detect stimulated electromagnetic emissions (SEE). The results of the experiment, given in the next section, show that SBS produces an SEE spectrum of electromagnetic waves with spectral lines that are offset from the pump frequency by the ion-acoustic frequency in the plasma. This interpretation is validated in Sect. 3 by a theoretical derivation of the wave matching conditions for producing SBS in magnetized plasmas like the ionosphere. The electric field amplitudes of the pump wave and the location of the upper-hybrid resonance region in the ionosphere are described in Sect. 4.

With the SBS matching conditions, the measured electromagnetic spectra are mapped to the plasma densities in the ionosphere. For vertical HF beams, the measured SBS emissions are shown to be consistent with electromagnetic waves at the region where the pump frequency is equal to the upperhybrid frequency. The matching conditions are applied to give a relation between the electron temperature and the SBS emission spectra. With this relation, the electron temperature in the modified ionosphere can be measured using the ionacoustic wave shifts in the SEE data along with knowledge of the electron and ion cyclotron frequencies at the ionospheric interaction heights. Thus the stimulated Brillouin spectra interpretation provides a powerful remote sensing tool for the modified ionospheric environment.

\section{Experimental measurements of Stimulated Brillouin Scatter using HAARP}

Electromagnetic backscattered waves were produced by the HAARP transmitter operating with pump frequencies of 4.5 and $5.6 \mathrm{MHz}$ with an effective radiated power (ERP) of 1.0 and 1.66 gigawatts, respectively. The time history of 
the waves was captured with a digital receiver called the GBOX5 using digital sampling and continuous recording of a $\pm 125 \mathrm{kHz}$ around the HF pump frequency. For the measurement of the stimulated electromagnetic emissions (SEE), an initial data sample time of 4 microseconds was used. Successive 600 points are averaged to provide data with a $2.4 \mathrm{~ms}$ sample period for the low-frequency spectral analysis. The stored in-phase and quadrature-phase (I and Q) data samples were processed to yield spectra of the backscatter wave sidebands around the pump wave center frequency with a resolution of $1.39 \mathrm{~Hz}$. The SEE spectrum is displayed in $\mathrm{dB}$ power relative to the maximum input power that will saturate the GBOX5 receiver. The input attenuation to the receiver was adjusted to prevent this saturation by the pump wave reflected from the ionosphere.

The GBOX5 recording system was located $20 \mathrm{~km}$ from the HAARP transmitter using a vertical pointing inverted V antenna with the nomenclature AS-2259/GR. This antenna is used as a near vertical incidence skywave antenna in the 3.5 to $10 \mathrm{MHz}$ frequency range. This lightweight, sloping dipole, omnidirectional antenna is ideal for SEE measurements because of its frequency range and portability. The same antenna was used for the SEE measurements of Norin et al. (2009).

The HAARP transmitter frequency was fixed at $4.5 \mathrm{MHz}$ in the time period between 19:40 to 20:00 UT (11:40 to 12:00 Local Time) on 24 October 2008. The $4.5 \mathrm{MHz}$ HF transmissions from HAARP ware generated in 120 s periods with $90 \mathrm{~s}$ on and $30 \mathrm{~s}$ off. The transmitter was either operated in a standard beam with all antenna elements in phase or in an orbital angular momentum configuration (OAM) with the antenna elements phase equal to their azimuthal position relative to the center of the array (Leyser et al., 2009). The OAM configuration produces a wider beam with a null along the beam axis. The GBOX5 receiver recorded data continuously through this transmission period.

A spectrogram of the recorded SEE was produced from successive $0.8 \mathrm{~s}$ data samples. The dynamic spectra recorded for a full power ( $1 \mathrm{GW}$ ERP) vertical-beam transmission at $4.5 \mathrm{MHz}$ is illustrated in Fig. 1. After a transient produced by the HAARP transmitter at turn on, the SEE spectra shows the central pump line, two stimulated Brillouin scatter lines around the pump and narrow lines at 120 and $180 \mathrm{~Hz}$. These latter features along with $60 \mathrm{~Hz}$ lines reported by Norin et al. (2009) are the result of the $60 \mathrm{~Hz}$ harmonics in the power supplies for the transmitter. The SBS lines were imbedded in a low-frequency continuum emission that may be produced as spectral noise by the HAARP transmitter. The HAARP transmitter transient that is shown as an initial broadening of the spectra in Fig. 1 has been reduced for future experiments.

The frequencies of the labeled lines in Fig. 2 were obtained by averaging the $90 \mathrm{~s}$ of spectral data. Each individual spectrum was obtained with a Blackman window of the data. The $120 \mathrm{~Hz}$ lines, produced by power supply ripple in the HAARP transmitter, are used to verify the frequency cali-

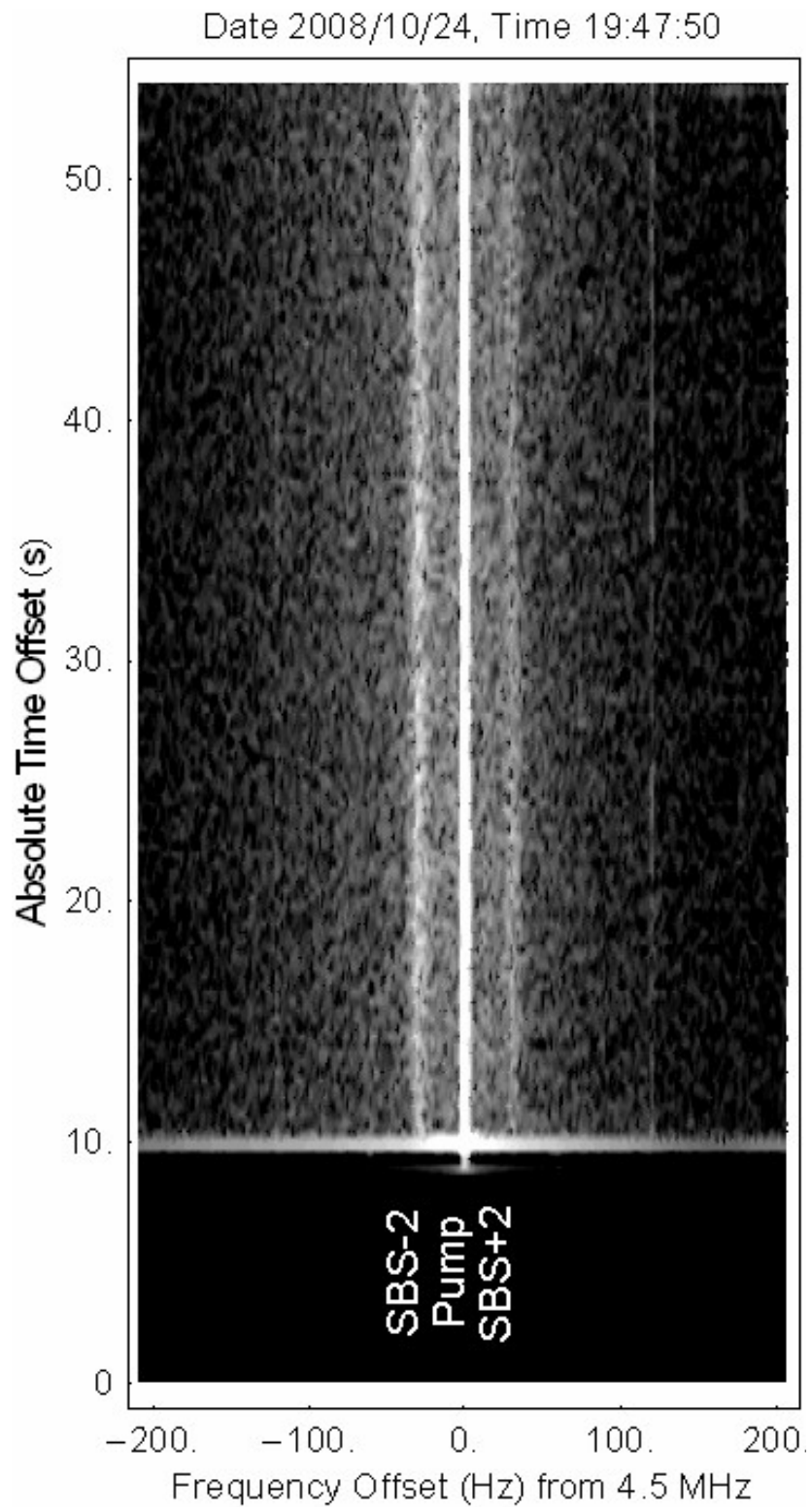

Fig. 1. Time history of the SEE spectra near the HF pump frequency of $4.5 \mathrm{MHz}$. The data were obtained on 24 October 2008 when the HF wave was turned on at 19:48 UT. The wave induced stimulated Brillouin scatter (SBS) lines near $30 \mathrm{~Hz}$ are broader than the artificial power line harmonics at 120 and $180 \mathrm{~Hz}$.

bration for the spectral measurements. The SBS lines near $30 \mathrm{~Hz}$ were determined to have frequency variations that distinguish them from a subharmonic of the $60 \mathrm{~Hz}$ power grid frequency. Table 1 shows the frequencies and power of the SBS spectra lines for each of the different modes pumped by $4.5 \mathrm{MHz}$. Spectral interpolation is used to give a measurement resolution of $0.7 \mathrm{~Hz}$. All of the downshifted lines are stronger than the upshifted lines (Fig. 2). Theoretical works on the stimulated Brillouin spectra in an unmagnetized plasma (Kruer, 1988; Eliezer, 2002) state that the production of the downshifted (or Stokes) lines should be preferred. 

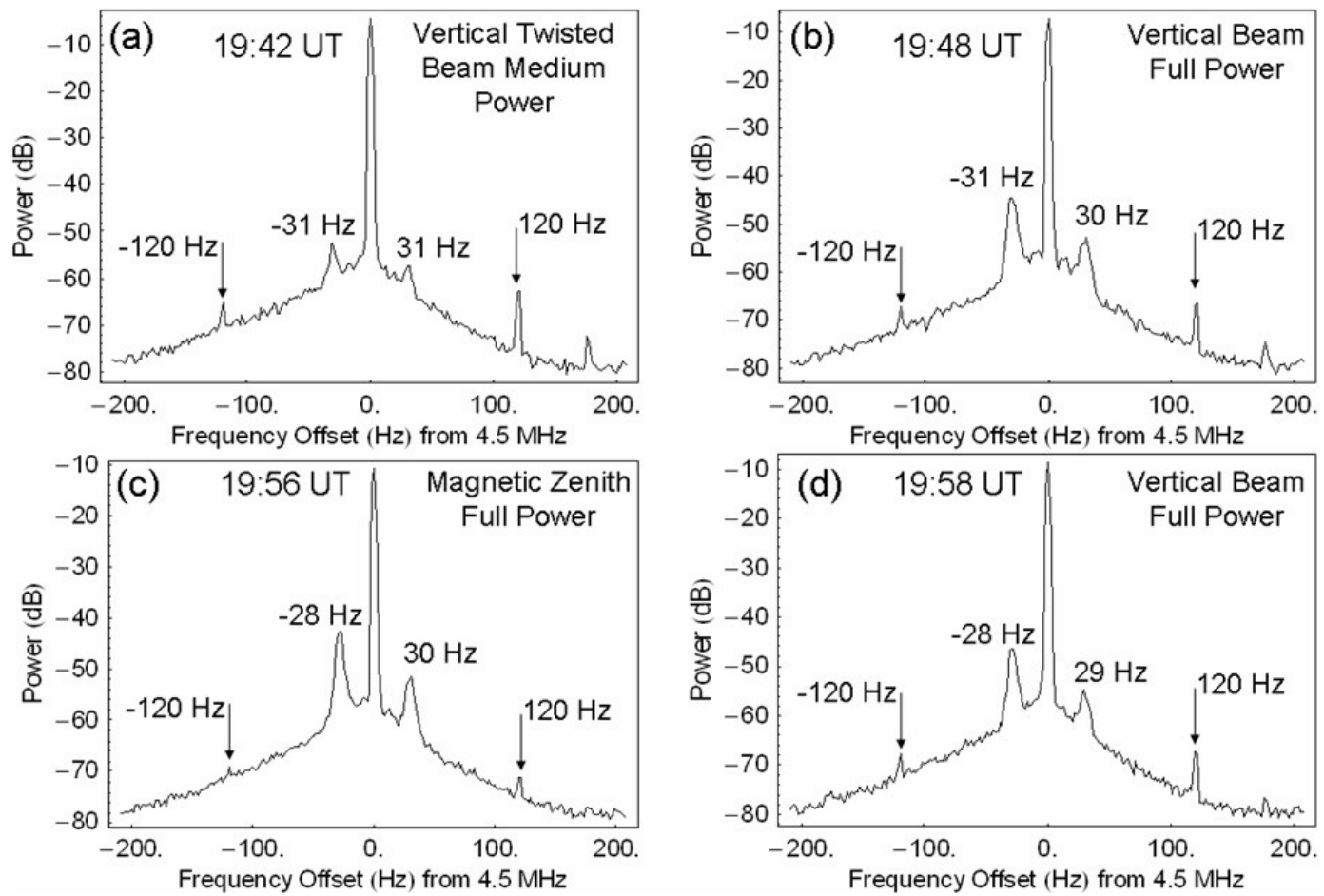

Fig. 2. Spectra of scattered electromagnetic waves from the HAARP transmitter operating at $4.5 \mathrm{MHz}$ with $1 \mathrm{GW}$ effective radiated power. All the data were taken within a $20 \mathrm{~min}$ period on 24 October 2008. The downshifted lines are also called the Stokes lines, downshifted SBS- lines or the downshifted narrow peaks (np-). The upshifted lines are similarly called the anti-Stokes lines, upshifted SBS+ lines or the upshifted narrow peaks (np+). The $120 \mathrm{~Hz}$ lines are produced by power ripple in the transmitter power system.

Table 1. SBS scattered electromagnetic line strengths and frequencies for a $4.5 \mathrm{MHz}$ pump wave.

\begin{tabular}{llccccc}
\hline Time & Mode & $\begin{array}{c}\text { Downshifted peak } \\
\text { frequency }\end{array}$ & $\begin{array}{c}\text { Upshifted peak } \\
\text { frequency }\end{array}$ & $\begin{array}{c}\text { Pump power } \\
(\mathrm{dB})\end{array}$ & $\begin{array}{c}\text { Downshifted line } \\
\text { power }(\mathrm{dB})\end{array}$ & $\begin{array}{c}\text { Upshifted line } \\
\text { power }(\mathrm{dB})\end{array}$ \\
\hline 19:42:00 & Medium vertical & $-30.6 \mathrm{~Hz}$ & $31.9 \mathrm{~Hz}$ & -4.5 & -52.5 & -57.4 \\
19:48:00 & Full power vertical & $-30.6 \mathrm{~Hz}$ & $30.6 \mathrm{~Hz}$ & -7.27 & -44.7 & -53.0 \\
$19: 56: 00$ & Full Magnetic Zenith & $-29.2 \mathrm{~Hz}$ & $29.9 \mathrm{~Hz}$ & -10.84 & -47.5 & -51.3 \\
$19: 58.00$ & Full power vertical & $-27.8 \mathrm{~Hz}$ & $29.2 \mathrm{~Hz}$ & -8.67 & -46.3 & -54.8 \\
\hline
\end{tabular}

Indeed, all of the SBS lines presented by Norin et al. (2009) also had stronger down shifted lines than the upshifted (anti-Stokes) lines. Norin et al. (2009) has similar displays of $\pm 30 \mathrm{~Hz}$ lines for vertical beams of HAARP HF waves. When the HF beam was pointed toward the magnetic zenith, however, the SBS spectra showed two sets of lines. One set was near $\pm 30 \mathrm{~Hz}$ as illustrated in Fig. 2c but in the same spectra there was a second set of lines near $\pm 15 \mathrm{~Hz}$ when the HF beam was aligned with the magnetic field lines. None of the measurements made at $4.5 \mathrm{MHz}$ showed this effect.

The stimulated Brillouin scatter (SBS) lines were observed using (1) a vertical HF beam in an orbital angular momentum (OAM) or twisted beam configuration with a transmit- ter power of $1.86 \mathrm{MW}$ (Fig. 2a), (2) full standard beam with 3.6 MW transmitter (Fig. 2b and d), as well as (2) a magnetic aligned standard beam with $3.6 \mathrm{MW}$ transmitter (Fig. 2c). All of the SBS lines were located very close to $\pm 30 \mathrm{~Hz}$ from the pump wave. The line frequency offsets shown in Fig. 2 are found as an average of the full $90 \mathrm{~s}$ when the HF was operating.

The OAM excitation of the ionosphere by HAARP has been previously demonstrated by Leyser et al. (2009). The spectra in Fig. 2a is the first reported excitation of SBS by an OAM beam. Mendonça, Thidé, and Then (2009) have suggested that the presence of OAM in the electromagnetic pump wave can affect the growth rate for the SBS instability. Our initial experiments at HAARP could not find any 
difference for excitation of SBS with the OAM beam or with a more-standard circularly polarized pump without orbitalangular-momentum. This study will be presented in a future paper.

The locations of the SBS lines were not significantly affected by either the shape and power of the pump wave or the tilting of the beam to the vertical or the magnetic zenith. Figure 2 is the first display of SBS lines at the same frequency, with a variety of beam configurations, and with a very short time period between each measurement. We find that tilting the HF beam or changing the beam shape has no measurable affect on the frequencies of the SBS lines relative to the fixed 4.5 $\mathrm{MHz}$ pump wave.

The Stokes (SBS-) and anti-Stokes (SBS+) lines are not exactly the same offset from the pump frequency. This low frequency offset near $30 \mathrm{~Hz}$ varies with time. The systematic variation is illustrated in Fig. 3 with the beam pointed toward the magnetic zenith. When the transmitter is turned on, the pump echo at $4.5 \mathrm{MHz}$ shows large fluctuations around an average of about $-10 \mathrm{~dB}$ on our relative scale. With the same scale, the power in the upshifted and downs shifted lines both decay by about $9 \mathrm{~dB}$ over a period of $30 \mathrm{~s}$.

The magnitude of the frequency shifts for the SBS lines relative to the pump are not the same when the data is examined in detail. The offset magnitude of the upshifted line is about $1 \mathrm{~Hz}$ or larger than the frequency offset for the downshifted line. As illustrated in Fig. 3, absolute value of the frequency offset for the upshifted line asymptotically changes with time reaching an equilibrium value in about $20 \mathrm{~s}$. This indicates that the SBS interactions in the plasma have slightly different conditions for the Stokes and anti-Stokes lines and these conditions for the weaker upshifted line are changing. This shift in the upshifted frequency offset probably results from Doppler shifts of the ionospheric reflected HF wave that scatters with ion-acoustic waves to produce the weaker upshifted, anti-Stokes line (see Sect. 4.). The frequency of the downshifted line does not change with time because the SBS occurs before reflection occurs.

For comparison, another sample of the SEE showing SBS lines is shown in Fig. 4. The SBS measurements at $5.6 \mathrm{MHz}$ used a 10-min long continuous wave transmission toward the magnetic zenith. The spectrum is dominated by a large downshifted line labeled SBS-1 located $16.7 \mathrm{~Hz}$ below the electromagnetic pump frequency. Two weak lines called $\mathrm{SBS}+1$ at $16.7 \mathrm{~Hz}$ and SBS+2 at $34.7 \mathrm{~Hz}$ are found above the pump frequency. From the data, it is not clear if the SBS+2 line is a cascade of $\mathrm{SBS}+1$ or a separate emission line. Norin et al. (2009) interpret the low frequency lines $(\mathrm{SBS} \pm 1)$ as originating near the reflection altitude and the $\mathrm{UH}$ resonance altitude as the source for the (SBS+2). All of the magnetic zenith SBS spectra provided by Norin et al. (2009) showed the strong downshifted lines near $17 \mathrm{~Hz}$.

The purpose of this paper is to provide a quantitative interpretation of the SBS spectra to measure (1) the electron temperatures in the heated plasma and (2) the profile of the
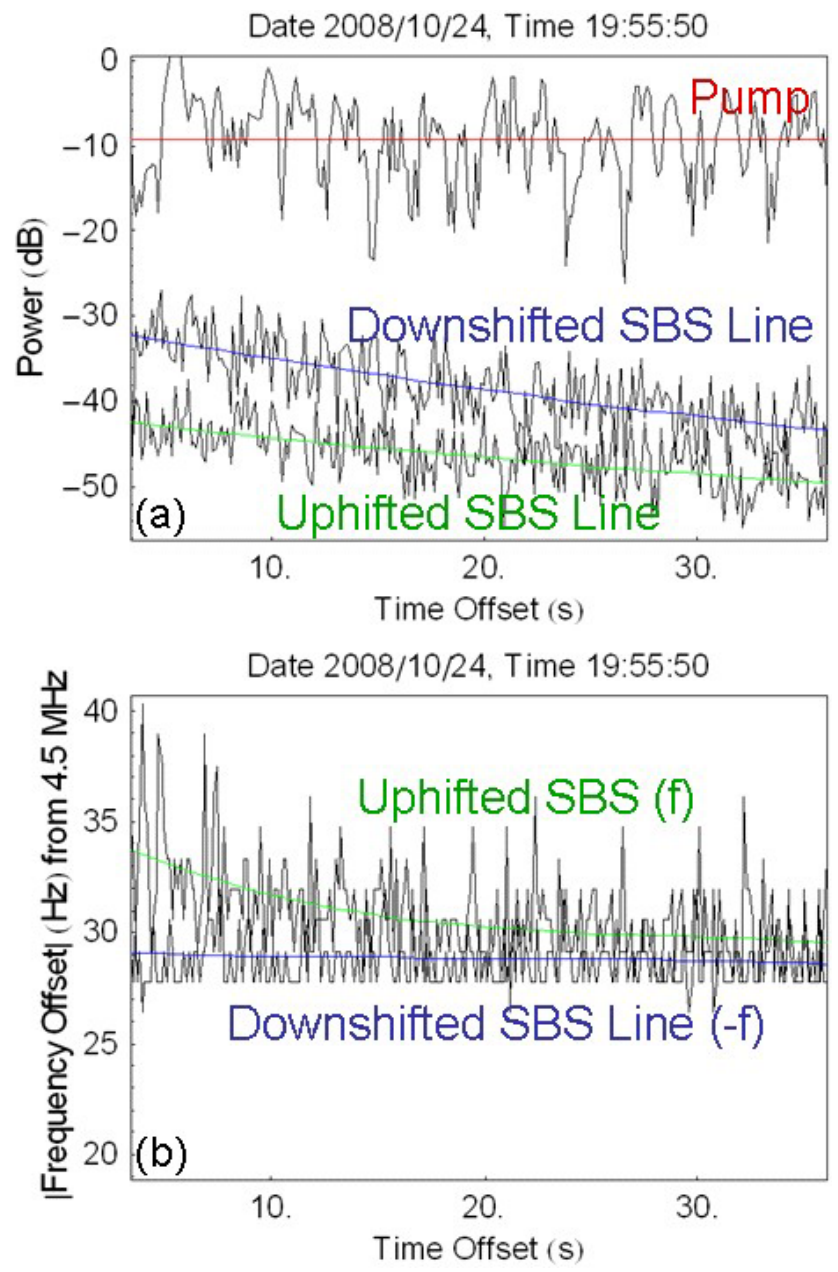

Fig. 3. Temporal variations in the (a) powers and (b) frequencies of the SBS line features. The average pump power stays nearly constant while the powers of both the upshifted and downshifted lines drop with time. The frequency of the pump remains fixed, but the offset of the upshifted SBS line drops from $34 \mathrm{~Hz}$ to about $30 \mathrm{~Hz}$ in $30 \mathrm{~s}$. The downshifted SBS line remains at about $29 \mathrm{~Hz}$ frequency.

scattered electromagnetic waves near both the reflection and the upper-hybrid resonance regions. For this interpretation, detailed knowledge of the background ionosphere, ambient electric field, and collisional absorption is required. Measurement of the ambient environment is discussed in the next section.

\section{Background environment during the SBS measurements}

To support the ionospheric modification experiments, the background plasma density profiles are measured with the HAARP digital ionosonde (digisonde) provided by the University of Lowell Massachusetts. The ionograms obtained 


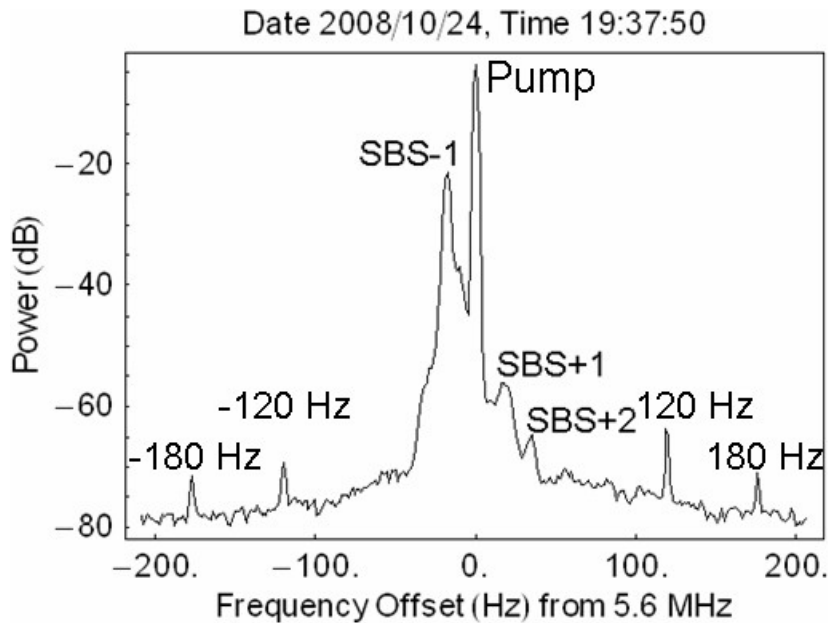

Fig. 4. Stimulated electromagnetic emission spectra for a $5.6 \mathrm{MHz}$ pump wave pointed toward the magnetic zenith from HAARP. A down shifted SBS-2 line may be masked by the low-frequency side of the strong SBS-1 line.

at 19:35 and 20:00 UT on 24 October 2004 were analyzed to provide true height profiles of the bottomside ionosphere. Linear interpolation of the ionogram data was used to provide the family of profiles illustrated in Fig. 5a. The plasma layers are stable below $170 \mathrm{~km}$ altitude over the $25 \mathrm{~min}$ of the ionosonde measurement. Above $170 \mathrm{~km}$, the F-layer densities are increasing causing a small amount of increase in the bottom side gradients between 170 and $190 \mathrm{~km}$ altitude. The ionogram data indicates that the F-region ionosphere was stable between 19:40 UT and 20:00 UT during the time of the high power radio wave experiments. The accurate electron density profile is essential to aid the determination of the altitude of the HF interactions in the plasma.

The magnetic field in the ionosphere is needed to determine the wave propagation characteristics of the magnetized plasma. The International Geomagnetic Reference Field (IGRF) model provided the magnetic field strength and direction in the upper atmosphere over HAARP. The magnetic field near the HF reflection altitude is estimated to be $|B|=5.205 \times 10^{-5}$ Tesla with a dip angle of 75.5 degrees.

The electron-ion and electron-neutral collision frequencies can affect the amplitude of the electromagnetic pump wave. The electron-ion collision frequency is calculated from the ion densities using the standard formula from Goldston and Rutherford (1995)

$v_{e i}=\frac{\sqrt{2} n_{i} e^{4} \ln \Lambda}{12 \pi^{3 / 2} \varepsilon_{0}^{2} \sqrt{m_{e}} T_{e}^{3 / 2}}$

where $n_{i}$ is the ion density (equal to the electron density from the ionosonde), $T_{e}$ is the electron temperature in energy units (Joules) and $\ln \Lambda=14$ in the ionosphere.

The NRL-MSISO0 model was used to estimate the neutral densities in the upper atmosphere over the HAARP transmit-

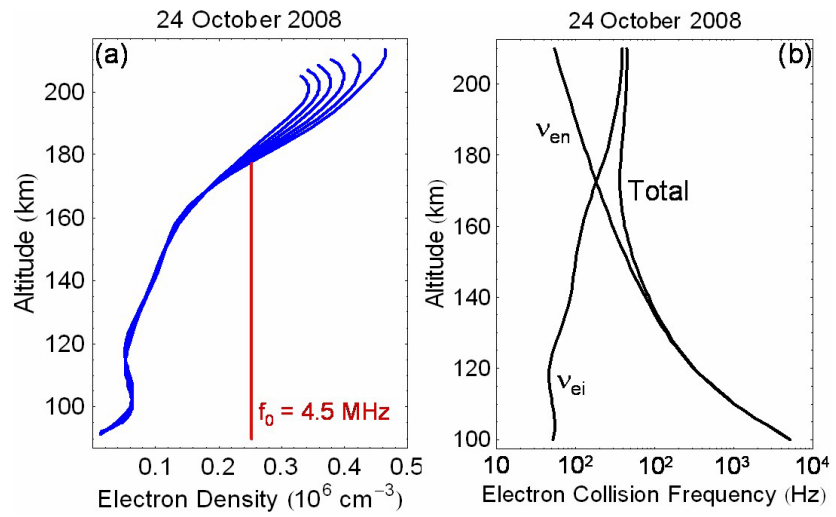

Fig. 5. Altitude profiles for (a) measured electron density profiles and $(\mathbf{b})$ estimated electron-neutral $\left(v_{e n}\right)$ and electron ion $\left(v_{e i}\right)$ collision frequencies for the period of the ionospheric modification experiments. Ionograms taken at 19:35 and 20:00 UT were analyzed and interpolated to provide the electron density profiles and electron-ion collision frequencies. The NRL-MSISO0 model provided the neutral densities for the estimates of $v_{e n}$.

ter. These densities are converted into electron-neutral collision frequencies by the formula from Yeh and Liu (1972)

$v_{\text {en }}=8.88 \times 10^{-5} \sqrt{T_{e}}\left(n_{\mathrm{O}_{2}}+n_{\mathrm{N}_{2}}+2 n_{\mathrm{O}}\right)$

where $T_{e}$ is the electron temperature (again in Joules), $n_{\mathrm{O}_{2}}, n_{\mathrm{N}_{2}}$ and $n_{\mathrm{O}}$ are the molecular oxygen, molecular nitrogen, and atomic oxygen densities in $\mathrm{m}^{-3}$, respectively and $v_{e n}$ is in $\mathrm{s}^{-1}$. The electron-neutral collision frequencies during the October Period of 2008 (Fig. 5b) are very low because of low neutral densities during the current solar minimum period. Near the reflection altitude, estimated value of $v_{e n}$ is $128 \mathrm{~s}^{-1}$ and $v_{e i}$ is $222 \mathrm{~s}^{-1}$ giving a total electron collision frequency of $v_{e T}=350 \mathrm{~s}^{-1}$. In addition, D-region absorption by electron neutral collisions during the daytime or during times of strong electron precipitation can significantly reduce the intensity of the pump wave before it interacts in the F-layer.

The next step is to derive a theory for stimulated Brillouin scatter (SBS) in a magnetized plasma. Previous work on SBS dealt with laser-plasma interactions where the background magnetic field plays an insignificant role in the SBS process. The basis for SBS is the parametric decay of an electromagnetic pump wave into an ion acoustic wave and a backscattered EM wave. The ionosphere has a strong enough magnetic field to affect the propagation of both electromagnetic and the ion-acoustic waves. The matching conditions for production of SBS are derived in the next section.

\section{Wave matching conditions for Stimulated Brillouin Scatter in magnetized plasmas}

Narrowband sidebands near the transmitted electromagnetic pump wave are thought to be produced by stimulated 
Brillouin Scatter (SBS) (Norin et al., 2009). The up-going electromagnetic pump wave at $\omega_{0}$ decays into (1) a highfrequency, down-going scattered electromagnetic wave at $\omega_{S}$ and (2) a low-frequency wave at frequency $\omega_{L}$. The wave frequency and wave propagation direction are given by the energy and momentum conservation equations

$\omega_{0}=\omega_{S}+\omega_{L}$

$\boldsymbol{k}_{0}=\boldsymbol{k}_{S}+\boldsymbol{k}_{L}$

where $\mathbf{k}_{0}, \mathbf{k}_{S}$, and $\mathbf{k}_{L}$ are the wave numbers for the upward electromagnetic pump, scattered electromagnetic wave and ion-acoustic wave, respectively (Kruer, 1988; Eliezer, 2002). This indicates that a photon or electromagnetic wave of one energy or frequency cannot produce a decay product with higher energy or frequency. Also the directional momentum of the decay products is conserved so that the decay products of an upward propagating wave must have at least one upward component.

Low frequency electrostatic waves are described by the dispersion equation for plasma waves as given by Eq. (4.20.3) of Yeh and Liu (1972).

$$
\begin{aligned}
1 & -\frac{X_{e}\left(1-Y_{e}^{2} \operatorname{Cos}^{2} \theta\right)}{1-Y_{e}^{2}-n_{L}^{2} \delta_{e}\left(1-Y_{e}^{2} \operatorname{Cos}^{2} \theta\right)} \\
- & \frac{X_{i}\left(1-Y_{i}^{2} \operatorname{Cos}^{2} \theta\right)}{1-Y_{i}^{2}-n_{L}^{2} \delta_{i}\left(1-Y_{i}^{2} \operatorname{Cos}^{2} \theta\right)}=0
\end{aligned}
$$

where the magneto-ionic parameters of normalized electron density, normalized gyro frequency, normalized thermal velocity, and refractive index are respectively defined as

$$
\begin{aligned}
X_{e, i} & =\frac{\omega_{p e, i}^{2}}{\omega_{L}^{2}}=\frac{N_{e, i} e^{2}}{m_{e, i} \varepsilon_{0} \omega_{L}^{2}}, Y_{e, i}=\frac{\Omega_{e, i}}{\omega_{L}} \\
& =\frac{e B}{m_{e, i} \omega_{L}}, \delta_{e, i}=\frac{\mathrm{v}_{T e, i}^{2}}{c^{2}}=\frac{\gamma_{e, i} T_{e, i}}{m_{e, i} c^{2}}, n_{L}=\frac{k_{L} c}{\omega_{L}} .
\end{aligned}
$$

and $T_{e, i}$ are the electron and ion temperatures in energy units. The angle $\theta$ is between the wave normal direction and the background magnetic field vector and it satisfies $\boldsymbol{B} \cdot \boldsymbol{k}_{L}=|\boldsymbol{B}|\left|\boldsymbol{k}_{L}\right| \operatorname{Cos} \theta$. For vertical propagation of both ion acoustic and electromagnetic waves through a horizontally stratified medium, $\theta$ is fixed at the complement of the magnetic dip angle over HAARP.

The ionosphere is assumed to be composed of positively charged oxygen ions with mass $m_{i}$ and electrons with mass $m_{e}$ with $m_{e} \ll m_{i}$. Also, the ion sound waves have wavelengths much larger than a Debye length so $k_{L} \lambda_{D} \ll 1$ where the Debye length is $\lambda_{D}=\sqrt{\frac{\gamma_{e} T_{e} \varepsilon_{0}}{N_{e} e^{2}}}$. Under these conditions, Eq. (4) simplifies to

$\omega_{L}^{4}-\left(\Omega_{i}^{2}+k_{L}^{2} c_{\mathrm{IA}}^{2}\right) \omega_{L}^{2}+\Omega_{i}^{2} k_{L}^{2} c_{\mathrm{IA}}^{2} \operatorname{Cos}^{2} \theta=0$

where $c_{\mathrm{IA}}=\sqrt{\frac{\gamma_{e} T_{e}+\gamma_{i} T_{i}}{m_{i}}}$ is the ion acoustic velocity with $\gamma_{e}=1$ and $\gamma_{i}=3$ (Stix, 1992). The solution of Eq. (6) for $k_{L}$ has a

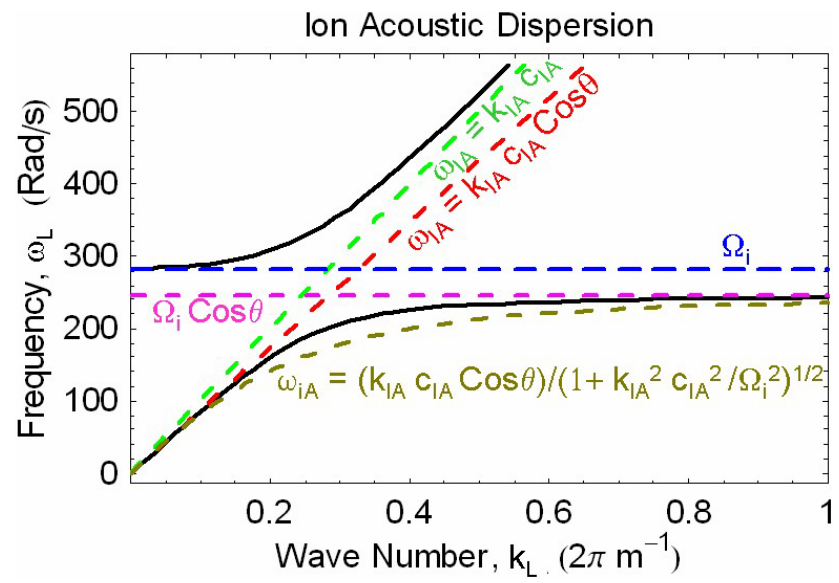

Fig. 6. Zeros, poles and limiting forms for the ion acoustic dispersion Eq. (6) near the ion gyro frequency. The black curve represents the most exact form used to compute the SBS matching conditions. Ion acoustic wave are found for frequencies below $\Omega_{i} \operatorname{Cos} \theta$ where $\theta$ is the wave normal angle relative to the magnetic field direction. Electrostatic ion cyclotron wave are found for frequencies just above the $\Omega_{i}$.

zero at $\omega_{L}=\Omega_{i}$ and a pole at $\omega_{L}=\Omega_{i} \operatorname{Cos} \theta$. This dispersion equation is illustrated in Fig. 6.

Two solutions to Eq. (6) are found for $\omega_{L}^{2}$ assuming that $\left|k_{L} c_{\mathrm{IA}}<\right| \Omega_{i} \mid$. For frequencies above $\Omega_{i}$, the electrostatic ion cyclotron (EIC) wave (Stix, 1992) is found with the frequency determined from

$$
\begin{aligned}
\omega_{\mathrm{EIC}}^{2} & =\frac{\Omega_{i}^{2}+k_{\mathrm{EIC}}^{2} c_{\mathrm{IA}}^{2}+\sqrt{\left(\Omega_{i}^{2}-k_{\mathrm{EIC}}^{2} c_{\mathrm{IA}}^{2}\right)^{2}+4 k_{\mathrm{EIC}}^{2} c_{\mathrm{IA}}^{2} \Omega_{i}^{2} \operatorname{Sin}^{2} \theta}}{2} \\
& \cong \Omega_{i}^{2}+\frac{k_{\mathrm{EIC}}^{2} c_{\mathrm{IA}}^{2} \operatorname{Sin}^{2} \theta}{1-k_{\mathrm{EIC}}^{2} c_{\mathrm{IA}}^{2} / \Omega_{i}^{2}} \text { if } k_{\mathrm{EIC}}^{2} c_{\mathrm{IA}}^{2} \ll \Omega_{i}^{2}
\end{aligned}
$$

The second low-frequency mode is the ion acoustic (IA) wave with the negative sign before the radical

$\omega_{\mathrm{IA}}^{2}=\frac{\Omega_{i}^{2}+k_{\mathrm{IA}}^{2} c_{\mathrm{IA}}^{2}-\sqrt{\left(\Omega_{i}^{2}+k_{\mathrm{IA}}^{2} c_{\mathrm{IA}}^{2}\right)^{2}-4 k_{\mathrm{IA}}^{2} c_{\mathrm{IA}}^{2} \Omega_{i}^{2} \cos ^{2} \theta}}{2}$

To second order in $\left|k_{\mathrm{IA}} c_{\mathrm{IA}}\right| /\left|\Omega_{i}\right|$, the ion acoustic wave dispersion is given as

$\omega_{\mathrm{IA}}^{2}=\frac{k_{\mathrm{IA}}^{2} c_{\mathrm{IA}}^{2} \Omega_{i}^{2} \cos ^{2} \theta}{\left(\Omega_{i}^{2}+k_{\mathrm{IA}}^{2} c_{\mathrm{IA}}^{2}\right)}$

This equation was used by Norin et al. (2009).

For ion acoustic frequencies much larger than the ion cyclotron frequency (i.e., $\omega_{\mathrm{IA}} \gg \Omega_{c i}^{2}$ ), Eq. (6) becomes

$\omega_{\text {IA }}^{2}-k_{\mathrm{IA}}^{2} c_{\mathrm{IA}}^{2}=0$.

This equation is used to describe the higher frequency ion acoustic waves produced by parametric decay of the electromagnetic pump into an electron plasma wave and an ion 


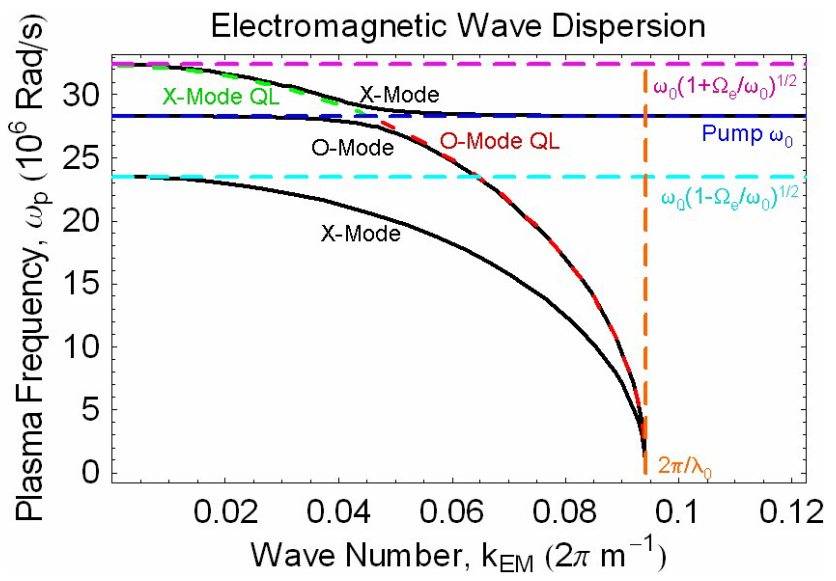

Fig. 7. Ionospheric plasma frequency for a $4.5 \mathrm{MHz}$ electromagnetic wave with a range of wave numbers extending past the freespace wave number.

acoustic wave for either a pump wave that is much larger than the plasma frequency (Fejer, 1979) or a plasma with zero magnetic field (Kruer, 1988).

Since neither Eq. (9) nor Eq. (10) are valid for lowfrequency modes near the ion gyro frequency, they will not be used here. The full dispersion Eq. (6) will be employed to determine the SBS matching conditions. Both ion acoustic waves and electrostatic ion-cyclotron waves are included in this dispersion equation. The full dispersion equation along with the approximate forms are illustrated by Fig. 6 for $c_{\mathrm{IA}}=1000 \mathrm{~m} / \mathrm{s}, \theta=15^{\circ}$, and $\Omega_{i}=283 \mathrm{rad} / \mathrm{s}(45 \mathrm{~Hz})$.

SBS involves two electromagnetic waves, one pump wave with subscript " 0 " and one scattered wave with subscript "s", that satisfy the Appleton-Hartree (A-H) equation. These waves can have O-Mode or X-Mode polarization as indicated by superscript "O" or "X", respectively. With Cartesian coordinates defined by Yeh and Liu (1972) such that the propagation vector is in the z-direction and the magnetic field vector is in the yz-plane, the transverse polarization is defined as $R(z)=E_{x} / E_{y}=-H_{y} / H_{x}$ for the transverse fields of each mode. The refractive index for these modes is given by Eq. (4.14.19) by Yeh and Liu (1972)

$n^{(O, X)}=\sqrt{1-\frac{X}{U+i Y R_{O, X} \operatorname{Cos} \theta}}$

where $n^{(O, X)}=k^{(O, X)} c / \omega$ is the O-Mode and X-Mode refractive index, $R^{(O, X)}$ is the wave polarization given by

$R^{(O, X)}(z)=\frac{i}{\operatorname{Cos} \theta}\left[\frac{Y \operatorname{Sin}^{2} \theta}{2(U-X)} \mp \sqrt{\frac{Y^{2} \operatorname{Sin}^{4} \theta}{4(U-X)^{2}}+\operatorname{Cos}^{2} \theta}\right]$,

the magnetoionic parameter definitions similar to those given in Eq. (5) with electromagnetic frequency $\omega$ and $U=1-i \frac{v_{e n}}{\omega}$ represents collisional loss between electrons and ions at rate $v_{e n}$. In terms of frequencies and wave numbers the electromagnetic wave dispersion equation $k^{2} c^{2}=n^{2} \omega^{2}$ for electromagnetic propagation in a magneto-ionic plasma is

$$
\begin{aligned}
\frac{2\left(\omega_{0, S}^{2}-\omega_{p}^{2}\right) \omega_{p}^{2}}{\omega_{0, S}^{2}-k_{0, S}^{2} c^{2}} & =\left(2\left(\omega_{0, s}^{2}-\omega_{p}^{2}\right)-\Omega_{e}^{2} \operatorname{Sin}^{2} \theta\right. \\
& \pm \sqrt{\left.\frac{4\left(\omega_{0, S}^{2}-\omega_{p}^{2}\right)^{2} \Omega_{e}^{2} \operatorname{Cos}^{2} \theta}{\omega_{0, S}^{2}}+\Omega_{e}^{4} \operatorname{Sin}^{4} \theta\right)}
\end{aligned}
$$

where the form is chosen so the + sign is used for the ordinary (O-Mode) propagation and the - sign for the extraordinary (X-Mode) propagation and the collisional loss term has been neglected. The low collision frequencies (Fig. 5b) have a negligible effect on the matching conditions but they will be re-introduced with computations of the amplitudes for the high power pump wave in the next section. For propagation approximately along $\mathbf{B}$ with $\theta \ll 1$, the quasi-longitudinal (QL) approximation of Eq. (13) is given by

$\omega_{p}^{2}=\left(\omega_{0, S}^{2}-k_{0, S}^{2} c^{2}\right)\left(1 \pm \operatorname{Sign}\left[\omega_{0, S}-\omega_{p}\right] \frac{\Omega_{e} \operatorname{Cos} \theta}{\omega_{0, S}}\right)$

but this equation is only valid if

$\operatorname{Cos}^{2} \theta \gg \frac{\omega_{0, S}^{2} \Omega_{e}^{2} \operatorname{Sin}^{4} \theta}{4\left(\omega_{p}^{2}-\omega_{0, S}^{2}\right)^{2}}$.

Near the plasma resonance region where the plasma frequency is close to the wave frequency the condition in Eq. (15) will break down. The relationship between the ionospheric plasma frequency and the electromagnetic wave number for propagation at $\omega_{0}=4.5 \mathrm{MHz}$ is shown in Fig. 7 . The magneto-ion conditions are the same as for Fig. 6 . The QL solutions are given by the red and green curves, respectively, for O-mode and X-mode propagation.

Since the SBS is expected to occur near to where the electromagnetic wave amplitude swells to a maximum at the critical layer near where $\omega_{p} \sim \omega_{0, S}$, the complete A-H formulation (Eq. 13) will be used for the full theoretical description. As illustrated in Fig. 7, the only mode that will propagate from the ground to the resonance region is the O-mode so the + sign in Eq. (13) is the most relevant to the model of the SBS matching conditions. The X-mode with the - sign in Eq. (13) also will be considered in the analysis for completeness because if the plasma layer is steep on the bottom side, some $\mathrm{X}$-mode energy may tunnel across the gap between the $\mathrm{X}$-mode cutoff at $\omega_{p}=\omega_{0}\left(1-\Omega_{e} / \omega_{0}\right)^{1 / 2}$ and the Z-mode resonance at $\omega_{p}=\omega_{0}\left(\omega_{0}^{2}-\Omega_{e}^{2}\right)^{1 / 2} /\left(\omega_{0}^{2}-\Omega_{e}^{2} \operatorname{Cos}^{2} \theta\right)^{1 / 2}$ (Yeh and Liu, 1972).

Relations (3), (6), and (13) provide five equations for the five unknowns $c_{\mathrm{IA}}, k_{0}, k_{S}, \omega_{L}$, and $k_{L}$ given the known quantities of pump wave frequency $\omega_{0}$, local plasma frequency $\omega_{p}$, electron and ion gyro frequency $\Omega_{e}$ and $\Omega_{i}$, and propagation angle $\theta$. These equations can be solved numerically to provide the SBS conditions. With the wave- number- squared 


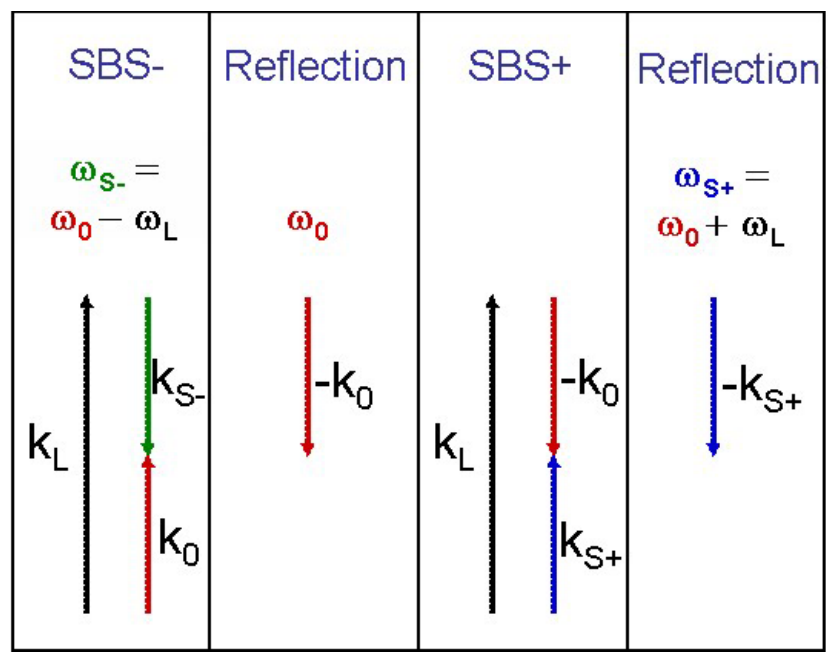

Fig. 8. Frequency and wave-number matching for generation of backscatter sidebands by stimulated Brillouin scattering (SBS). The direction of pump and upward scattered electromagnetic waves are reversed by ionospheric reflection for reception on the ground.

parameters in dispersion Eqs. (6) and (13), the signs are chosen to be opposite for $k_{0}$ and $k_{S}$ to yield a low frequency wave number $k_{L}$ that has a magnitude equal to $\left|k_{0}-k_{S}\right|$ in agreement with Eq. (3).

Strictly speaking, stimulated Brillouin Scatter (SBS) is only the decay of electromagnetic waves into ion-acoustic waves and scattered electromagnetic waves. Classic treatments of SBS have only considered unmagnetized plasmas (Kruer, 1988). As mentioned in reference to Eq. (6), in the ionosphere when a magnetic field is imposed on the plasma the electrostatic ion cyclotron wave may also be excited by the SBS-like process. For the following discussions about Fig. 8, a generalized SBS is discussed in relation to excitation of the ion-acoustic wave found below the ion-cyclotron frequency and the electron ion cyclotron waves just above the ion-cyclotron frequency as shown in Fig. 6. Consequently, $k_{L}$ and $\omega_{L}$ are used to represent the wave number and frequency of all low-frequency decay products.

The wave number diagrams in Fig. 8 illustrate the SBS matching conditions. The pump frequency decay in Eq. (3) indicates a loss of EM energy from the pump to generate a low frequency ion acoustic wave. The low frequency $\omega_{L}$ is greater than zero and the downward scattered electromagnetic wave $\omega_{S_{-}}=\omega_{0}-\omega_{L}$ is downshifted from the pump wave. The downshifted SBS- line is called the Stokes line that has a lower energy than the pump line. The resultant scattered electromagnetic wave vector is negative (or downward) with a value given by $\mathbf{k}_{S-}=\mathbf{k}_{0}-\mathbf{k}_{L}$ since both $\mathbf{k}_{0}$ and $\mathbf{k}_{L}$ are upward vectors and $\left|\mathbf{k}_{0}\right|<\left|\mathbf{k}_{L}\right|$ as shown in Fig. 8. After reflection, downward pump wave $\left(\omega_{0},-k_{0}\right)$ can split into a down going ion acoustic away and an upward propagating scattered EM wave. After reflection, the upward EM wave is transformed into a downshifted, down-going EM wave that adds to the scattered wave amplitude. Consequently, the wave vectors in the first panel of Fig. 8 could be reversed to represent the generation of SBS- by the reflected pump wave.

Next, the upshifted SBS+ emission is explained. The upward pump will reflect in the ionosphere near where refractive index goes to zero yielding a downward pump wave with wave vector $-\mathbf{k}_{0}$. This reflected wave will scatter from the ion-acoustic waves to yield an upward scattered electromagnetic wave with wave vector $\mathbf{k}_{S+}=\mathbf{k}_{0}+\mathbf{k}_{L}$ at frequency $\omega_{S+}=\omega_{0}+\omega_{L}$. The scattered upshifted electromagnetic wave will propagate upward until it reflects in the ionosphere above the generation region. This wave with wave number $-\mathbf{k}_{S+}$ will propagate downward to be received on the ground as an upshifted anti-Stokes sideband of the pump wave. The SBS generation of both the Stokes and antiStokes lines is illustrated in Fig. 8. The anti-Stokes line will have contributions from both the upgoing and downgoing, reflected pump wave.

Using Fig. 8, the Doppler shift of the anti-Stokes line (Fig. 3b) can be explained as a wave reflected by a moving reflector. For non-relativistic motion, the amount of Doppler shift $(\Delta f)$ for scatter from a body moving with velocity $(\mathbf{v})$ is given by $\Delta f=f \mathbf{v} ?\left(\mathbf{k}_{0}^{s}-\mathbf{k}_{0}^{i}\right) / c$ where $f$ is the incident frequency, $\mathbf{k}_{0}^{s}$, and $\mathbf{k}_{0}^{i}$ are unit vectors in the direction of the scattered and incident waves, respectively (Ruck et al., 1970). As illustrated in Fig. 8, the pump wave generates the downshifted SBS emissions without reflection and, consequently, any motion of the reflection altitude does not show up in the Stokes line. This constancy of the downshifted frequency is exactly what is shown by the blue line in Fig. $3 \mathrm{~b}$.

From Fig. 8, it is shown that the upshifted line is driven by the reflected pump wave. If the medium is moving downward with velocity $V$, the frequency of the Dopplershifted reflected wave is $\omega_{0}+2 V \omega_{0} / c$. After scattering with the ion acoustic waves at frequency $\omega_{\mathrm{IA}}$, the electromagnetic wave propagates upward and is reflected by the ionosphere. The resulting upshifted wave has a frequency of $\omega_{0}+\omega_{\text {IA }}+4 V \omega_{0} / c$. Note that the Doppler shift of the reflected pump wave is one-half that of the upshifted SBS line. The initially measured $5 \mathrm{~Hz}$ frequency offset corresponds to a Doppler motion of about $80 \mathrm{~m} / \mathrm{s}$ in the HF reflection altitude. When the pump wave is first turned on, electron heating seems to yield a lowering in the HF reflection altitude. The positive Doppler shift from this reduction in the altitude of reflection is consistent with an initial enhancement in the plasma density near the reflection altitude. As the plasma approaches thermal equilibrium, this Doppler shift in the SBS+ line is reduced to nearly zero.

The following theory applies to both the electrostatic ion cyclotron and ion-acoustic waves. Because the scattered and pump EM wave frequencies are almost equal, the matching conditions equation is well approximated by letting $\mathbf{k}_{s} \cong-\mathbf{k}_{0}$ 


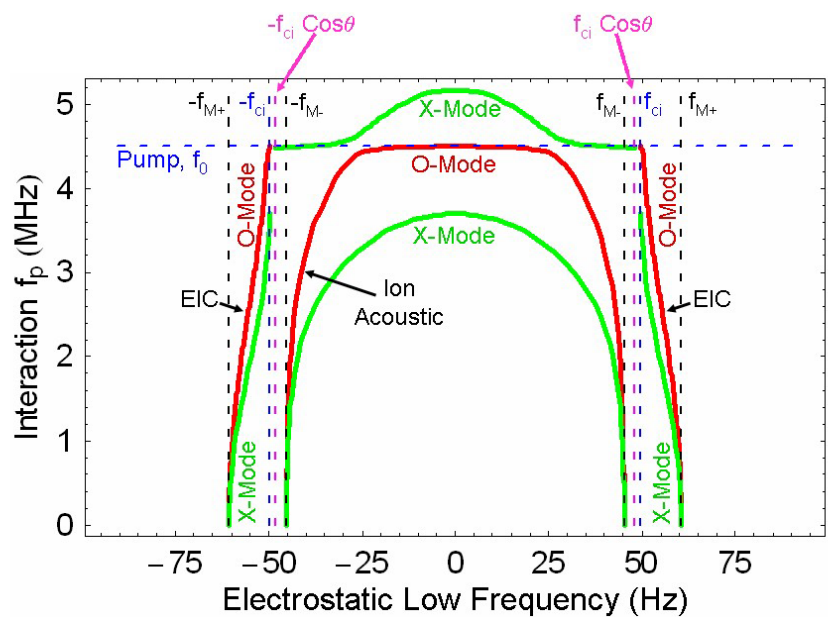

Fig. 9. Generalized SBS matching conditions for O-Mode and XMode electromagnetic waves at $4.5 \mathrm{MHz}$ for an ionosphere with an ion sound speed of $1600 \mathrm{~m} / \mathrm{s}$ and an ion gyro frequency $f_{c i}=49.6 \mathrm{~Hz}$ propagating at an angle of $14.5^{\circ}$ with respect to magnetic field direction.

so $\mathbf{k}_{L} \cong 2 \mathbf{k}_{0}$. This is substituted into Eq. (6) and solved to yield either an equation for the ion sound frequency

$\omega_{L}^{2}=\frac{1}{2}\left(4 c_{\mathrm{IA}}^{2} k_{0}^{2}+\Omega_{i}^{2} \pm \sqrt{16 c_{\mathrm{IA}}^{4} k_{0}^{4}+\Omega_{i}^{4}-8 c_{\mathrm{IA}}^{2} k_{0}^{2} \Omega_{i}^{2} \operatorname{Cos} 2 \theta}\right)$

where the + sign represents the EIC wave and the - sign denotes the IA wave. In either case the ion sound speed is

$c_{\mathrm{IA}}^{2}=\frac{\omega_{L}^{2}}{4 k_{0}^{2}} \frac{\Omega_{i}^{2}-\omega_{L}^{2}}{\Omega_{i}^{2} \operatorname{Cos}^{2} \theta-\omega_{L}^{2}}$

where $k_{0}^{2}$ is given by Eq. (13). With prescribed values of wave frequency, propagation angle, plasma frequency, electron and ion cyclotron frequencies in the interaction region, Eqs. (13) and (16) provides an accurate description of the relation between the ion sound speed and two possible values of low frequency waves excited by generalized SBS process. The limits to the ion sound speeds are found by substituting the free space value $\omega_{0} / c$ for $k_{0}$ in Eq. (16). Those frequencies are

$$
\begin{aligned}
\omega_{M \pm}^{2}= & \frac{1}{2}\left(\frac{4 c_{\mathrm{IA}}^{2} \omega_{0}^{2}}{c^{2}}+\Omega_{i}^{2}\right. \\
& \left. \pm \sqrt{\frac{16 c_{\mathrm{IA}}^{4} \omega_{0}^{4}}{c^{4}}+\Omega_{i}^{4}-\frac{8 c_{\mathrm{IA}}^{2} \omega_{0}^{2} \Omega_{i}^{2} \operatorname{Cos} 2 \theta}{c^{2}}}\right) .
\end{aligned}
$$

An analytic solution to the matching equations is derived to yield insight into the SBS interactions in magnetized plasmas. The quasi-longitudinal (QL) approximations to the electromagnetic wave dispersion equations are given by Eq. (14). This is combined with Eqs. (16) and (3) to give the plasma frequency at the SBS interaction altitude

$\omega_{p}^{2}=\omega_{0}\left(\omega_{0} \pm \Omega_{e} \operatorname{Cos} \theta \operatorname{Sign}\left[\omega_{0}^{2}-\omega_{p}^{2}\right]\right)$

$$
\left(1-\frac{c^{2} \omega_{\mathrm{IA}}^{2}}{4 c_{\mathrm{IA}}^{2} \omega_{0}^{2}} \frac{\left(\Omega_{i}^{2}-\omega_{0}^{2}\right)}{\Omega_{i}^{2} \operatorname{Cos}^{2} \theta-\omega_{0}^{2}}\right)
$$

remembering that this equation is only valid if the QL conditions (Eq. 15) are satisfied.

The matching conditions depend on the wave frequency, the plasma frequency and the ion-sound speed at the interaction region. In the ionosphere, the generalized SBS can occur at a range of electron densities corresponding to the matching condition plasma frequency. The ion-sound speed will be determined by the electron and ion temperatures that are elevated above ambient conditions by the electromagnetic pump wave. For electron temperatures in the range of 1000 to $4000 \mathrm{~K}$ and ion temperatures between 600 and $1000 \mathrm{~K}$ the ion-sound speed $c_{\text {IA }}$ takes values from 1200 to $1900 \mathrm{~m} / \mathrm{s}$. For fixed ion-sound speeds, Eqs. (12) and (16) are used to numerically compute the matching plasma frequency $f_{p e}$ as a function of the matching electrostatic low frequency $f_{L}$. For the same parameters, if the QL approximation is satisfied, Eq. (19) will provide an analytic expression of the plasma frequency corresponding to the local plasma density in the interaction region.

The computed matching conditions for the HAARP experimental parameters are graphed in Fig. 9 based on numerical computations with Eqs. (13) and (16). Electrostatic ion cyclotron solutions are found for frequencies just above the ion cyclotron frequency $f_{c i}$. The ion acoustic wave solution is limited to frequencies less than the longitudinal component $\left(f_{c i} \operatorname{Cos} \theta\right)$ of the ion cyclotron frequency. The plasma frequency of the SBS interaction is determined by the electron density profile. Figure 9 illustrates that both O-mode and Xmode pump waves can excite the SBS instability. For each ion sound speed there are three values of plasma frequency that provide matching, one below the pump frequency for $\mathrm{O}$ mode, one below the pump frequency for X-Mode, and one above the pump frequency for X-mode. The QL approximation expression (18) does not include this effect but passes through the $f_{0}=f_{p}$ boundary as the sign is changed from + for O-Mode to - for X-mode. This paper only presents data for O-Mode excitation but future experiments at HAARP will attempt to excite SBS by X-Mode waves.

To generate Fig. 9, the vertical pump wave at $4.5 \mathrm{MHz}$ is assumed to propagate with an angle of 14.46 degrees with the background magnetic field. The electron and ion gyro frequencies are given by the IGRF magnetic field for the October 2008 time period. The values representative of the conditions over HAARP at $178 \mathrm{~km}$ altitude are $f_{c e}=1.457 \mathrm{MHz}$ and $f_{c i}=49.6 \mathrm{~Hz}$, respectively. The SBS matching conditions are computed for a warm ionosphere with an ion sound speed of $1600 \mathrm{~m} / \mathrm{s}$. The QL-approximation to the matching conditions works well except where the wave frequency $f_{0}$ becomes close to the local plasma frequency $f_{p}$. The results using the full numerical solution to Eqs. (3), (6) and (13) are indistinguishable in Fig. 9 from the solutions using Eqs. (13) and (16). 
The low frequency solutions from O-mode interactions are restricted to the ranges $0<f_{\mathrm{IA}}<f_{M-}$ and $f_{c i}<f_{\mathrm{EIC}}<f_{M+}$ where $f_{M-}=45.2 \mathrm{~Hz}$ and $f_{M+}=60.6 \mathrm{~Hz}$ in this example. Only stimulated electromagnetic emissions in the ionacoustic frequency range have been measured. With these frequency limits, the low-frequency continuum that extends out to $200 \mathrm{~Hz}$ in Fig. 2 cannot be produced by SBS. The next step in the theory is to determine the plasma frequencies of the regions where the electromagnetic pump interacts with the plasma.

\section{The electromagnetic pump wave in the ionosphere}

The HAARP HF transmissions are generated with a ground based phased array which employ $360 \times 10 \mathrm{~kW}$ transmitters operating continuously. The pump signal at $4.5 \mathrm{MHz}$ had an effective radiated power of $P_{\mathrm{Eff}}=1 \mathrm{GW}$. The electric fields in the ionosphere are a standing wave composed of the upward wave from the transmitter and the reflected down-going wave. The electric field of the upward wave is related to the time averaged Poynting vector as

$$
\frac{P_{\mathrm{Eff}}}{4 \pi z^{2}}=\frac{|E|^{2} n}{\eta_{0}}
$$

where $z$ is the altitude in meters, $\mathrm{n}$ is the refractive index, and $\eta_{0}=377 \mathrm{Ohms}$ is the impedance of free space.

The structure of the standing wave in the ionosphere is determined by the background electron density profile. From the profiles in Fig. 5 for the electron densities for 19:58 UT on 24 October 2008, it was determined that the $4.5 \mathrm{MHz}$ pump wave reflected in the ionosphere at the critical density of $2.51 \times 10^{5} \mathrm{~cm}^{-3}$ at an altitude $\left(z_{0}\right)$ of $177.74 \mathrm{~km}$.

The amplitudes of the high power electric fields are computed using the wave equations for vertical propagation in a horizontally-stratified, fluid plasma with a steady magnetic field. These equations were first derived by Försterling (1942) and described in great detail by Budden $(1969,1985)$, Yeh and Liu (1972), and Lundborg and Thidé $(1985,1986)$. The second order differential equation for O-mode propagation is given as

$$
\frac{\partial^{2} F^{(O)}(z)}{\partial z^{2}}+k^{(O)}(z)^{2} F^{(O)}(z)=0
$$

where $k_{0}$ is found from Eq. (11) including the collisional absorption terms. Coupling to a similar equation for the extraordinary X-mode is neglected with the smooth gradients in the F-layer profile (Yeh and Liu, 1972; Lundborg and Thidé, 1985,1986 ). The electron density profile is used to compute a $k_{0}^{2}$ with transition from positive to negative values across the resonance altitude $z_{0}$. Below this altitude, the pump wave propagates upward and then reflects at $z_{0}$ to produce the down-going wave. Above the plasma resonance altitude, the O-mode wave becomes evanescent and quickly decays. Both numerical solutions (e.g., Budden, 1969) and approximate analytical solutions (see Lundborg and Thidé, 1985, 1986) have been used to compute solutions to Eq. (21). In this paper, the finite difference approximations to the second derivative in Eq. (21) provide the basis for the numerical solutions.

The boundary conditions above and below the resonance altitude are specified by WKB solutions of the form

$$
F_{\mathrm{WKB}}^{(O)}(z)=F^{(O)}\left(z_{1}\right) \sqrt{\frac{k^{(O)}\left(z_{1}\right)}{k^{(O)}(z)}} \exp \left[-i \int_{z_{1}}^{z} k^{(O)}\left(z^{\prime}\right) d z^{\prime}\right]
$$

where $z_{1}$ is a reference altitude where the electric field amplitude is specified (Yeh and Liu, 1972). The three electric field components of the wave are related to the function $F^{(O)}(z)$ by the equations

$$
\begin{aligned}
& \left\{E_{x}(z), E_{y}(z), E_{z}(z)\right\} \\
& \quad=\left\{1, R^{(O)}(z)^{-1}, R_{L}^{(O)}\right\} \frac{F^{(O)}(z)}{\sqrt{R^{(O)}(z)^{-2}-1}}
\end{aligned}
$$

where the subscripts $x, y$ and $z$ denote the magnetic east, north and up directions, $R^{(O)}(z)$ is the O-mode polarization in Eq. (12) and $P_{L}^{(O)}(z)$ is the O-mode longitudinal polarization given by Eq. (4.14.22) of Yeh and Liu (1972) as

$R_{L}^{(O)}(z)=\frac{i Y \operatorname{Sin} \theta}{(U-X)}\left[1-n^{(O)}(z)^{2}\right]$

The transverse polarization $(\mathrm{R}(\mathrm{X}, \mathrm{O}))$ is the ratio $E_{x} / E_{y}$ of the transverse electric fields. The longitudinal polarization $R_{L}^{(O)}(z)$ is the ratio $E_{z} / E_{x}$ of the longitudinal to northward fields. The O-Mode and X-Mode polarizations range from circular $\left(R^{(X, O)}= \pm i\right)$ near the reflection point to linear $\left(R^{(X, O)}=\infty\right.$ or 0$)$ near the reflection point. The longitudinal polarization is only large near the reflection point. The electric field component along the direction of the magnetic field is give by $E_{\|}(z)=E_{z}(z) \operatorname{Cos} \theta-E_{y}(z) \operatorname{Sin} \theta$ where $\theta$ is again the complement of the magnetic dip angle. Excellent descriptions of these features are provided by Budden (1966, 1985), Yeh and Liu (1972), and Lundborg and Thidé (1985, 1986).

The numerical solution to Eq. (21) starts at altitude $z_{1}=0.97 z_{0}=172.4 \mathrm{~km}$ with an upward propagating wave with a transverse electric field of $1.29 \mathrm{~V} / \mathrm{m}$ corresponding to $1 \mathrm{GW}$ ERP at $4.5 \mathrm{MHz}$ in the ionosphere neglecting any D-region absorption. The computation is terminated at the altitude $z_{2}=1.01 z_{0}=179.5 \mathrm{~km}$ where the wave is strongly damped in the overdense plasma layer. Pump wave reduction by mode conversion on field aligned irregularities at the UH resonance altitude is not included in the computation. The plasma frequency profile and the computed horizontal/transverse $\left(E_{x}\right.$, $\left.E_{y}\right)$ and vertical/logitudinal $\left(E_{z}\right)$ electric fields are illustrated in Fig. 10.

The vertical component just below the reflection altitude is much larger than the horizontal components of the wave. 


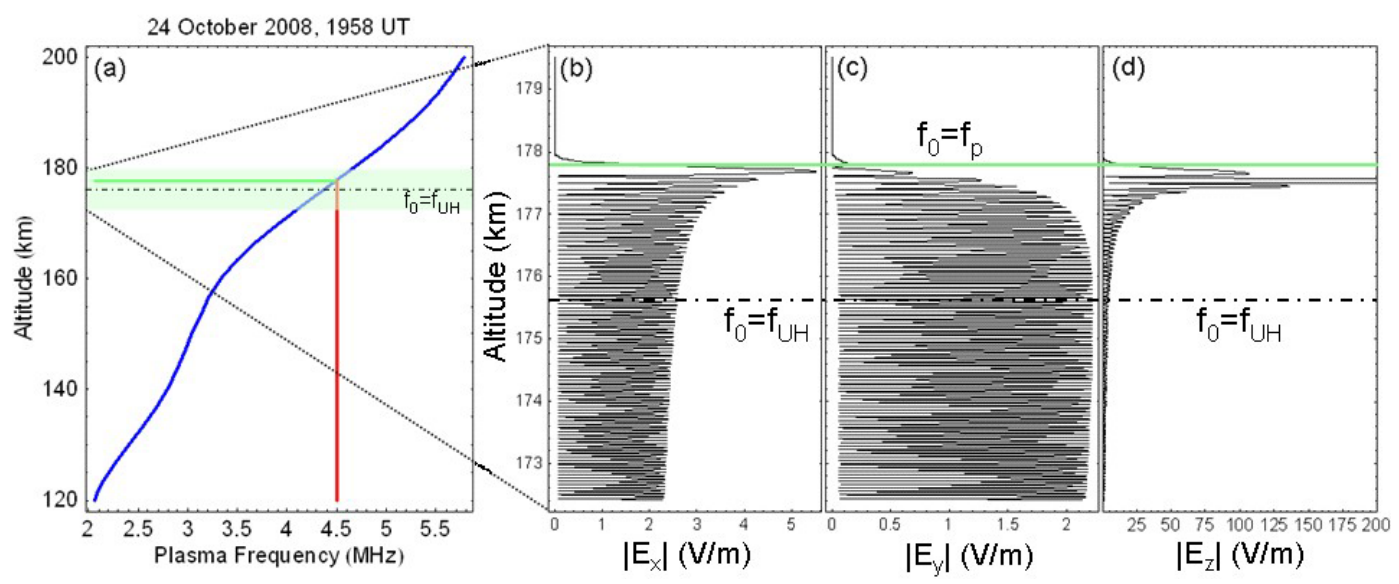

Fig. 10. Plasma frequency profile (a) and computed transverse (b, c) and longitudinal (d) electric fields produced by the $4.5 \mathrm{MHz}$ HAARP transmitter. The maximum electric field is found in the longitudinal component $E_{z}=671 \mathrm{~V} / \mathrm{m}$ just below the plasma resonance at $177.74 \mathrm{~km}$ altitude where the local plasma frequency is $4.48 \mathrm{MHz}$. For reference, the altitude where the wave frequency matches the upper hybrid frequency is shown with the horizontal dot-dashed line.

Similar effects have been computed by Lundborg and Thidé $(1985,1986)$ using the uniform approximation technique. The peak transverse field is $5.5 \mathrm{~V} / \mathrm{m}$ at $177.7 \mathrm{~km}$ altitude, $0.04 \mathrm{~km}$ below the plasma resonance altitude. The peak vertical/longitudinal electric field is $671 \mathrm{~V} / \mathrm{m}$ at $177.55 \mathrm{~km}$, $0.19 \mathrm{~km}$ below the plasma resonance altitude. For such large field strengths, the plasma will be strongly modified and the linear dispersion equations used to describe the matching conditions will no longer be valid. Outside this narrow region, the electric fields are on the order of a few $\mathrm{V} / \mathrm{m}$ and the matching equations remain valid. The computation used 10000 points along the vertical axis and the simulation code was checked using know analytic Airy function solutions to linear variations in $k_{0}^{2}$.

The details of the three electric field components near the reflection altitude are given in Fig. 10b, c, and d. The magnetic east component $E_{x}$ and the magnetic north component $E_{y}$ are transverse to the direction of propagation. Their amplitudes increase as the reflection altitude is approached but the upward longitudinal component along the direction of propagation has the largest electric field (Fig. 10d). This extremely large longitudinal field could easily couple into longitudinal ion sound waves and a down-going longitudinal component of an O-Mode electromagnetic wave.

The wave matching conditions used in the previous section use both frequency and wave number matching. The standing wave ripples illustrated by Fig. 10 shows that at the upper hybrid resonance level the wave number (or inverse wavelength) is well defined with upgoing and downgoing waves producing electric field pattern. Near the reflection height were the pump wave grows to over $670 \mathrm{~V} / \mathrm{m}$, the single peak is not well represented by a wave number. In this region, a full nonlocal treatment is needed to determine the coupling between high frequency EM waves and the low frequency electrostatic wave. A full wave treatment of this process will be explored in a future paper.

Soon after the pump wave is turned on, field aligned irregularities form in the F-layer and interactions at these irregularities absorb the wave energy to prevent pump EM wave from reaching the reflection altitude. Many of the SEE observations have led to the interpretation that much of the EM pump wave energy is deposited near the upper hybrid level where the wave frequency is equal to the upper hybrid frequency (Leyser, 2001). The horizontal dashed line in Fig. 10 provides the location of the upper hybrid altitude $\left(z_{\mathrm{UH}}=174.6 \mathrm{~km}\right)$ where $\omega_{0}^{2}=\omega_{\mathrm{UH}}^{2}=\omega_{p}^{2}+\Omega^{2}$ using a pump frequency of $4.5 \mathrm{MHz}$ and an electron gyro frequency of $1.457 \mathrm{MHz}$. This value of $f_{c e}$ was based on the magnetic field from the IGRF model. At the upper hybrid altitude, the pump wave has both transverse and longitudinal components of comparable amplitudes. For the simulation illustrated in Fig. 10, the peak electric fields near the UH resonance altitude are $\left(E_{x}, E_{y}, E_{z}\right)=(2.22,2.49,2.33) \mathrm{V} / \mathrm{m}$. The next section examines whether interactions near the plasma wave reflection altitude or near the upper hybrid resonance altitude produce the SBS emission lines.

The effects of pump reduction by coupling to upper hybrid waves have not been considered in the production of Fig. 10. The actual amplitude of the pump wave will have a first maximum just below level where upper hybrid waves are produced by mode conversion on field-aligned-irregularities. A second maximum will occur just below the plasma-frequency resonance altitude where the group velocity of the wave goes to zero and the amplitude peaks. The received SEE spectra may be used to estimate the strength of the scattered electromagnetic waves at these two levels. 


\section{Altitude profiles of the scattered electromagnetic waves for vertical beams}

In this section, the ion-acoustic SBS matching conditions will be used to determine the altitude of the observed scattered EM waves for vertical propagation in a horizontally stratified ionosphere. It will be shown that the fields driving the instability can be the large longitudinal electric fields near the plasma frequency resonance altitude or the transverse and longitudinal electric field components near the upper hybrid resonance altitude. Since all the observed frequency offsets of the scatter electromagnetic waves are less than the ion cyclotron frequency, the possible presence of electrostatic ion cyclotron waves will not be considered in this section. Consequently, the subscript IA will be used in place of the subscript $L$ to designate ion acoustic frequencies and wave numbers.

The scattered EM wave is offset by the pump wave by the ion acoustic frequency according to Eq. (3). All of the ion-acoustic offsets for the scattered EM wave illustrated in Figs. 2 and 4 are less than the ion cyclotron frequency of $49.6 \mathrm{~Hz}$ at the reflection altitude. To map the ion acoustic frequencies determined by subtracting the pump frequency from the scattered frequency, then Eq. (17) is rearranged to give the electromagnetic wave number

$k_{0}^{2}=\frac{\omega_{\mathrm{IA}}^{2}}{4 c_{\mathrm{IA}}^{2}} \frac{\Omega_{i}^{2}-\omega_{\mathrm{IA}}^{2}}{\Omega_{i}^{2} \operatorname{Cos}^{2} \theta-\omega_{\mathrm{IA}}^{2}}$

and that wave number is used in Eq. (13) to give the plasma frequency at the SBS interaction region. The mapping curves between the ion acoustic frequencies and the ionospheric plasma frequency are given in Fig. 11 for the HAARP experiment parameters using a range of ion-sound speeds. Since the ion acoustic frequencies for interactions at the UH levels from the HAARP SEE data are between 27 and $32 \mathrm{~Hz}$, the solutions with temperatures corresponding to an ion sound speed less than $1500 \mathrm{~m} / \mathrm{s}$ are ruled out. The two interaction levels where (1) the wave frequency is slightly less than the local plasma frequency or $\omega_{0}=\omega_{p}$ and (2) the wave frequency equals the local upper hybrid frequency are $\omega_{0}^{2}=\omega_{\mathrm{UH}}^{2}=\omega_{p}^{2}+\Omega^{2}$ are indicated on Fig. 11 by horizontal dashed lines. The upper hybrid level is given by $\omega_{p}=\left(\omega_{0}^{2}-\Omega^{2}\right)^{1 / 2}=26.75 \times 10^{6} \mathrm{rad} / \mathrm{s}$ or $4.26 \mathrm{MHz}$. The upper hybrid matching level is important because the pump wave strongly interacts with the plasma at this level in conjunction with field aligned irregularities produced by the HF beam (Leyser, 2001).

The black dashed curves in Fig. 11 show the QL solution (19) for the same conditions. The QL approximation to the O-mode matching conditions for SBS is usable near the point $\left(f_{\mathrm{UH}}\right)$ where the wave frequency is equal to the local value of the upper hybrid frequency. The QL curves are asymptotic to the full solution for each ion-acoustic speed in regions below the reflection height where $f_{0}=f_{p}$.

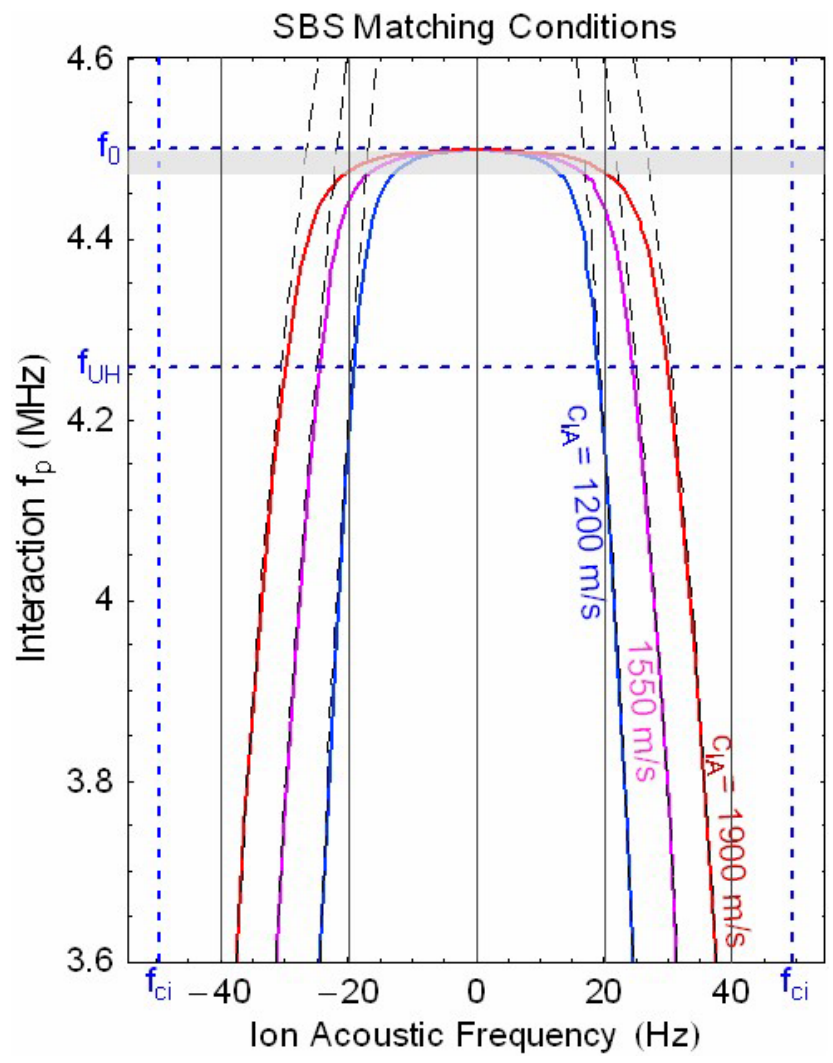

Fig. 11. SBS matching conditions for the O-mode pump at 4.5 MHz. The measured SBS lines with $29 \mathrm{~Hz}$ ion-acoustic offsets (Fig. 2) are consistent with an ion acoustic speed of $1900 \mathrm{~m} / \mathrm{s}$. The black dashed lines are the QL solution for the matching conditions for both O-mode and X-mode. The shaded portion of the figure shows where the strongest longitudinal wave can excite ion acoustic waves by SBS. The frequency $f_{\mathrm{UH}}$ denotes plasma frequency were the wave frequency is equal to the upper hybrid frequency.

Figure 11 shows that if the SBS excitation of the scattered EM waves occurs close to but just below the plasma frequency resonance at $f_{0}$, the ion acoustic frequency will be less than $20 \mathrm{~Hz}$. The peak longitudinal pump amplitude in Fig. 10c occurs at a plasma frequency of $4.48 \mathrm{MHz}$. At this frequency, the SBS matching curve shown in Fig. 11 gives an ion acoustic frequency of $17 \mathrm{~Hz}$ using an ion-acoustic speed of $1900 \mathrm{~m} / \mathrm{s}$. This is much smaller than any of the ion-acoustic offsets recorded in the SEE measurements of Figs. 1 and 2. Such small frequencies were reported by Norin et al. (2009) and are seen in Fig. 4 when the HF beam was tilted toward the magnetic zenith. The matching conditions diagram shows that all $17 \mathrm{~Hz}$ SBS must originate near the HF reflection altitude, not near the UH region. All of the interactions at the UH level have ion-acoustic frequencies on the order of $30 \mathrm{~Hz}$. For the $30 \mathrm{~Hz}$ waves to be produced near the reflection altitude, the matching conditions would give an unrealistically large ion-acoustic speed computed by the matching conditions at $3280 \mathrm{~m} / \mathrm{s}$ for an impossible electron 


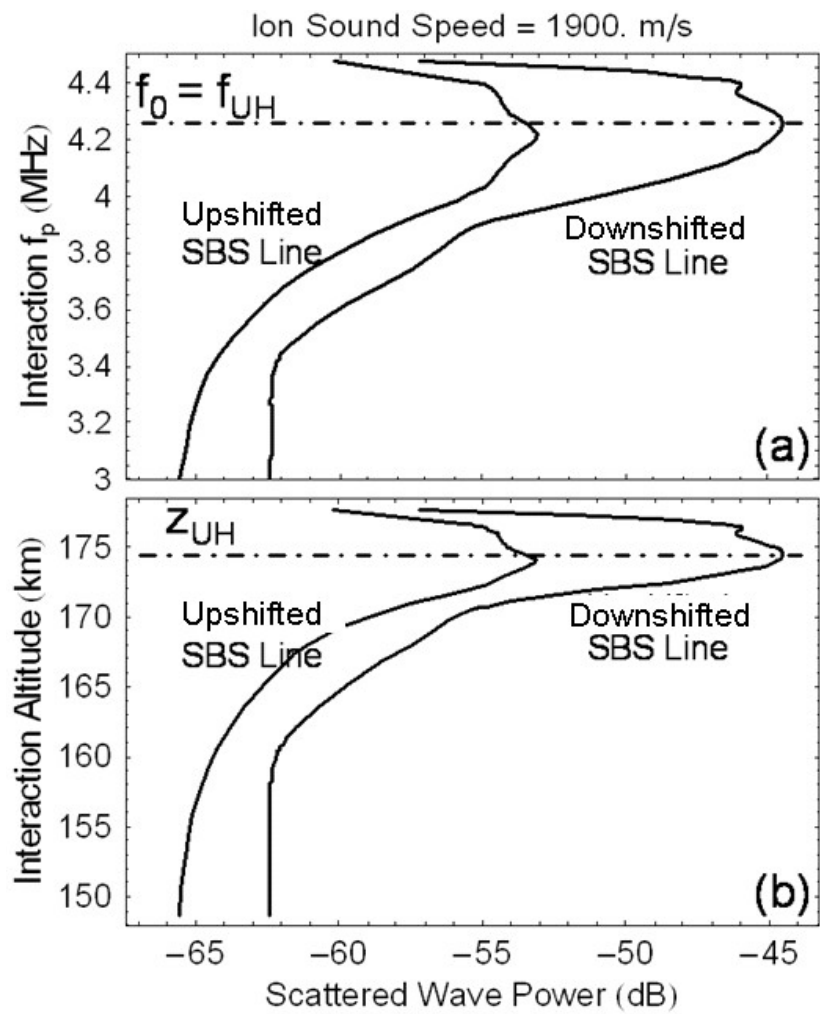

Fig. 12. Reconstructed profiles of scattered O-mode waves in the ionosphere. The upshifted and downshifted SBS ion-acoustic lines from Fig. 2c are first mapped to (a) the plasma frequency using the matching conditions for SBS with the assumption of a $1900 \mathrm{~m} / \mathrm{s}$ ion sound speed. The measured electron density profiles in Fig. 5 relate the local plasma frequency to the altitude yielding (b) the altitude profile of scattered EM wave power. The maximum scattered wave coincides with the location of the upper hybrid resonance.

temperature of $11500 \mathrm{~K}$. Any changes in the ion-acoustic speed at the UH level become manifested as changes in the ion-acoustic frequency offset of the SBS EM wave.

The next step is to use the map between ion acoustic frequency and interaction plasma frequency to produce a profile of the scattered wave power in the ionosphere. For downshifted and upshifted SBS lines shown in Fig. 2a, the matching conditions yield the scattered wave power profiles shown in Fig. 12. The plasma frequency profile is determined using the mapping function with $c_{\mathrm{IA}}=1900 \mathrm{~m} / \mathrm{s}$. This ionacoustic speed provides a good match between the measured ion-acoustic peak frequency and the SBS matching condition curve. The altitude profile is obtained with electron density profile at 19:48 UT from Fig. 5 where the electron density is converted to plasma frequency. This plasma-frequency profile determination depends on the assumptions that (a) the heated electron and ion temperatures give an ion sound speed of $1900 \mathrm{~m} / \mathrm{s}$ and (b) that the electron density profile has not been modified by the HF facility to significantly distort the values given in Fig. 5.
The top of Fig. 12 is just below the reflection altitude where the pump wave fields shown in Fig. 10 reach their maximum. With the assumed $1900 \mathrm{~m} / \mathrm{s}$ ion-sound speed, the peak scattered fields are very close to the altitude of the upper hybrid wave resonance with the pump wave. This mapping shows a broad region around the UH altitude for scattering of the EM pump wave by SBS. The concept is that the EM pump amplitude is depleted over the large a 6-km region near the UH resonance altitude before approaching the reflection altitude. The ion acoustic speed parameter, $c_{\mathrm{IA}}$, can be adjusted to place the scattered wave peak at the UH altitude. Similarly, lowering the sound speed to $1887 \mathrm{~m} / \mathrm{s}$ brings the peak of the downshifted SBS profile to the UH level. Near the UH level, the matching curves in Fig. 11 are more vertical than near the reflection altitude. The estimated value for ionsound speed is insensitive to variation in altitude around the UH level. The SBS instability seems to be occurring near the $\mathrm{UH}$ altitude $\left(z_{\mathrm{UH}}\right)$ were the wave frequency is equal to the local upper hybrid frequency. At this altitude, it has been postulated that the electromagnetic pump wave decays into upper hybrid and lower hybrid waves and that the upper hybrid waves mode converts to an electromagnetic wave to be received on the ground as the downshifted maximum (DM) in the spectrum of stimulated electromagnetic emissions (SEE) (e.g. Leyser, 2001). The results of Fig. 12 suggest that, for high enough power pump wave, the SBS instability is also excited around the UH level to produce the observed SBS spectral lines in the SEE.

If the SBS emissions come from the UH level, a simple expression for the ion sound speed can be derived. The QL approximation given by Eq. (19) is valid at the UH matching altitude. The UH level is defined by the condition $\omega_{0}^{2}-\Omega_{e}^{2}=\omega_{p}^{2}$. Substituting this into Eq. (19) for the O-mode and solving for $c_{\text {IA }}$ yields.

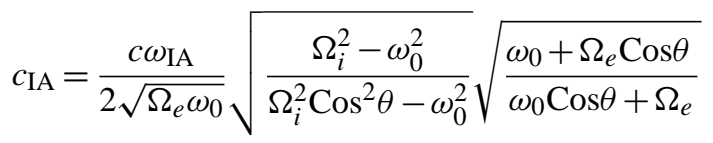

At 19:56 UT, the measured upshifted ion acoustic frequency is $f_{\mathrm{IA}}=29.2 \mathrm{~Hz}$ or $\omega_{\mathrm{IA}}=183.5 \mathrm{rad} / \mathrm{s}$. (Note: Frequency in $\mathrm{Hz}$ is only used for comparison to the measured frequency offsets in the scattered SBS lines). The computed electron and ion cyclotron frequencies are $\Omega_{e}=9.16 \times 10^{6} \mathrm{rad} / \mathrm{s}$, $\Omega_{i}=312 \mathrm{rad} / \mathrm{s}$ and magnetic field angle from vertical $\theta=14.5^{\circ}$ based on the magnetic field components from the IGRF model at $175 \mathrm{~km}$ altitude. With these parameters and using the pump frequency $f_{0}=4.5 \mathrm{MHz}$ or $\omega_{0}=28.3 \times 10^{6} \mathrm{rad} / \mathrm{s}$, Eq. (26) gives an ion sound speed of $c_{\mathrm{IA}}=1812 \mathrm{~m} / \mathrm{s}$. Similarly the downshifted ion acoustic frequency of $-30 \mathrm{~Hz}$ yields an ion sound speed of $1865 \mathrm{~m} / \mathrm{s}$. These values are both about $2.5 \%$ lower than those using the more accurate formulas (13) and (16).

The electron temperature can be found by using the definition of the ion sound speed after Eq. (6) and assuming that 
Table 2. Electron temperatures determined from Stimulated Brillouin Scattering for vertical heating of the ionosphere at $4.5 \mathrm{MHz}$.

\begin{tabular}{lcccc}
\hline Time $(\mathrm{UT})$ & \multicolumn{2}{c}{$19: 48$} & \multicolumn{2}{c}{$19: 58$} \\
Line & SBS - & SBS+ & SBS - & SBS + \\
\hline$f_{\mathrm{IA}}(\mathrm{Hz})$ & -30.6 & 30.6 & -29.2 & 27.8 \\
$C_{\mathrm{IA}}(\mathrm{m} / \mathrm{s})$ & 1780 & 1780 & 1690 & 1580 \\
$T_{e}(\mathrm{~K})$ & 3500 & 3500 & 3180 & 2870 \\
\hline
\end{tabular}

$T_{e}=3 T_{i}$ which is a sufficient condition for prevention of Landau damping of ion acoustic waves.

$T_{e}=\frac{m_{i} c^{2} \omega_{\mathrm{IA}}^{2}}{\left(\gamma_{e}+\gamma_{i} / 3\right) 4 \Omega_{e} \omega_{0}} \frac{\Omega_{i}^{2}-\omega_{0}^{2}}{\Omega_{i}^{2} \operatorname{Cos}^{2} \theta-\omega_{0}^{2}} \frac{\omega_{0}+\Omega_{e} \operatorname{Cos} \theta}{\omega_{0} \operatorname{Cos} \theta+\Omega_{e}}$

Table 2 gives the values for ion-acoustic speed and electron temperature obtained from Eqs. (26) and (27), respectively, using the ion acoustic frequency data of Table 1 and the other parameters previously given for the HAARP experiment. Only the vertical beam observations are used because theory was developed for vertical incidence on a horizontally stratified layer. The changes in the electron temperatures show a drop of about $500 \mathrm{~K}$ for the $10 \mathrm{~min}$ interval between the two HF experiments. With an estimated accuracy of $0.7 \mathrm{~Hz}$ for the SBS frequencies, the uncertainty for the recovered ion sound speed is $\Delta c_{\mathrm{IA}}=50 \mathrm{~m} / \mathrm{s}$ and for electron temperature is $\Delta T_{e}=200 \mathrm{~K}$. The assumption of $T_{e}=3 T_{i}$ makes the ions sound speed determination more reliable than the electron temperature measurements. This $T_{e}$ determination can be improved by considering the thermal coupling between the electrons and ions. The factors that cause the apparent electron temperature to drop over a period of $10 \mathrm{~min}$ are (1) change the coupling to the plasma by variations in the altitude profile of the F-layer or (2) change in the effective heating by increased D-region absorption. These processes that affect $T_{e}$ should be studied in the future with physics based models.

The measurement technique embodied in Eqs. (26) and (27) should be robust against variations in the actual SBS production efficiency. Even if the ion-acoustic waves are heavily Landau damped (when $T_{e} \sim T_{i}$ ) or damped by ionneutral collisions, the SBS instability can proceed if the scattered pump wave is not strongly damped (Kruer, 1988; Eliezer, 2002). With damped ion-acoustic waves, the SBS is local but growing. The threshold conditions for the SBS instability in a magnetized plasma needs to be developed to fully understand the pump wave fields needed to produce the low-frequency SEE observations.

\section{SBS matching at the magnetic zenith}

The examples considered thus far for SBS measurements were made with a vertical beam in a horizontally stratified

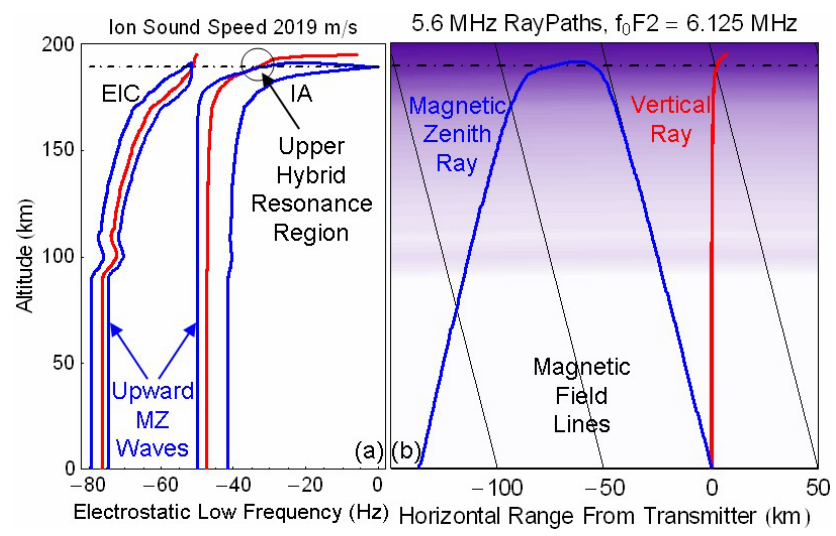

Fig. 13. Ion acoustic (IA) and Electrostatic Ion Cyclotron (EIC) wave matching frequencies (a) and ray paths (b) for the $5.6 \mathrm{MHz}$ wave propagating to the vertical (red) and to the magnetic zenith. When the paths of either ray cross the upper hybrid resonance region, nearly the same ion-acoustic frequency can be excited.

ionosphere. If the HF beam is tilted off vertical, the wave normal angle relative to the magnetic field direction will vary with altitude. Raytracing in the anisotropic plasma can provide both the wave number magnitude $k_{0}$ and the wave normal angle $\theta$. These quantities can be substituted in Eq. (17) to give the relationship between the ion sound speed and the ion acoustic frequency along the ray propagation path.

The Hamilton's equations for ray paths using the refractive index in a magnetized plasma (Eq. 13) are given by Hazelgrove (1955), Yeh and Liu (1972) and Budden (1985). These equations were solved numerically for propagation in the measured ionosphere over the HAARP transmitter. The OMode raytrace results for $5.6 \mathrm{MHz}$ are illustrated in Fig. 13. The electron density is given by the purple shading on the right. The vertical ray shown in red keeps the same wave normal angle $\theta$ throughout the propagation because the magnetic field direction is nearly constant with altitude relative to the vertical wave-propagation direction. The physical path bends slightly to the north near the reflection altitude.

The magnetic zenith (MZ) path starts out along the magnetic field direction with a propagation angle of zero. This angle stays near zero except within a few kilometers below the plasma resonance altitude where the angle becomes 90 degrees at reflection. The wave propagation angle $\theta$ at the upper hybrid resonance altitude is 12 degrees for the upgoing $\mathrm{MZ}$ ray. The ray propagates horizontally for about $10 \mathrm{~km}$ with a wave normal nearly perpendicular to $B$. The ray returns to earth with a 28 degree angle relative to $B$. With this MZ ray path, no upshifted SBS line would be produced by the $\mathbf{k}$-vector matching process illustrated in Fig. 8 because the downgoing ray is not in the region of the upward ion acoustic waves. The observations of upshifted SBS lines indicate the presence EM waves with simultaneous upward and downward propagation along the same field-aligned path. 
The finite width of the beam may allow scattering of displaced downward EM waves from the center by ion-acoustic waves excited by high power EM waves at the edge.

Two sets of matching conditions are illustrated in the left panel of Fig. 13. The electron ion cyclotron (EIC) wave can yield waves in the 50 to $70 \mathrm{~Hz}$ range. These waves have not been detected in the observations. The set of matching conditions for frequency offsets of $50 \mathrm{~Hz}$ or less come from the ion acoustic waves. These are the waves recorded by ground SEE measurements. At the upper hybrid altitude the SBS matching conditions (Eq. 17) are nearly identical for both the vertical and MZ ray paths along the upward trajectories (Fig. 13 left). For those rays and the same electron temperatures or ion sound speeds, the ion acoustic frequencies for the SBS-2 lines should be nearly identical. This may account for the consistency in the downshifted SBS peak frequencies given by Table 1 .

The upshifted SBS line for vertical pump waves shows evidence of Doppler motion of the ionospheric reflection surface. As illustrated by the temporal variations in Fig. 3 and discussed in Sect. 4, the reflected pump and scattered EM waves combine to produce upshifted SBS lines with slightly higher than the offsets of the downshifted SBS lines. A slight reduction of the ionosphere reflection level may occur just after the high power HF by a localized region of enhanced ionization. The reflected EM waves are then Doppler shifted by this lowering in the reflection altitude. Future experiments should investigate the initial shifts in the SBS lines to confirm this observation.

With the wave number and propagation angle provided by raytracing, the electron temperature measurement technique can be extended for use at regions where the SBS is excited just below the pump reflection altitude at the magnetic zenith. During the time period around 19:30 UT on 24 October 2008, the HAARP transmitter was operated at full power to provide $1.66 \mathrm{GW}$ at $5.6 \mathrm{MHz}$. The measured SEE spectra during that time showed strong SBS-1 lines with an downshifted peak at $-14.5 \mathrm{~Hz}$ and two upshifted SBS lines SBS+1 at $17.5 \mathrm{~Hz}$ and SBS+2 at $36.0 \mathrm{~Hz}$ as previously shown by Fig. 4. The downshifted SBS line from the UH altitude region is probably masked by the strong downshifted SBS- 1 emission.

To support the $5.6 \mathrm{MHz}$ observations, rays were propagated with the measured electron density profile given in Fig. 5. With the raytracing solution, the values of O-Mode wave number $k_{0}$ and the propagation angle $\theta$ are used with Eq. (16) to compute the ion acoustic frequency that satisfies the SBS matching conditions. As with $4.5 \mathrm{MHz}$, the $5.6 \mathrm{MHz}$ pump wave provides nearly identical ion acoustic wave frequencies at the upper hybrid resonance altitude. Compared to the $4.5 \mathrm{MHz}$ results, there is a larger band of ion-acoustic waves that satisfy the SBS matching conditions in a band between -10 to $-20 \mathrm{~Hz}$. This may account for the large spread in the downshifted SBS line as illustrated in Fig. 4.

The wave-matching conditions for the $5.6 \mathrm{MHz}$ pump were computed using the theory of the Sect. 3 neglecting the

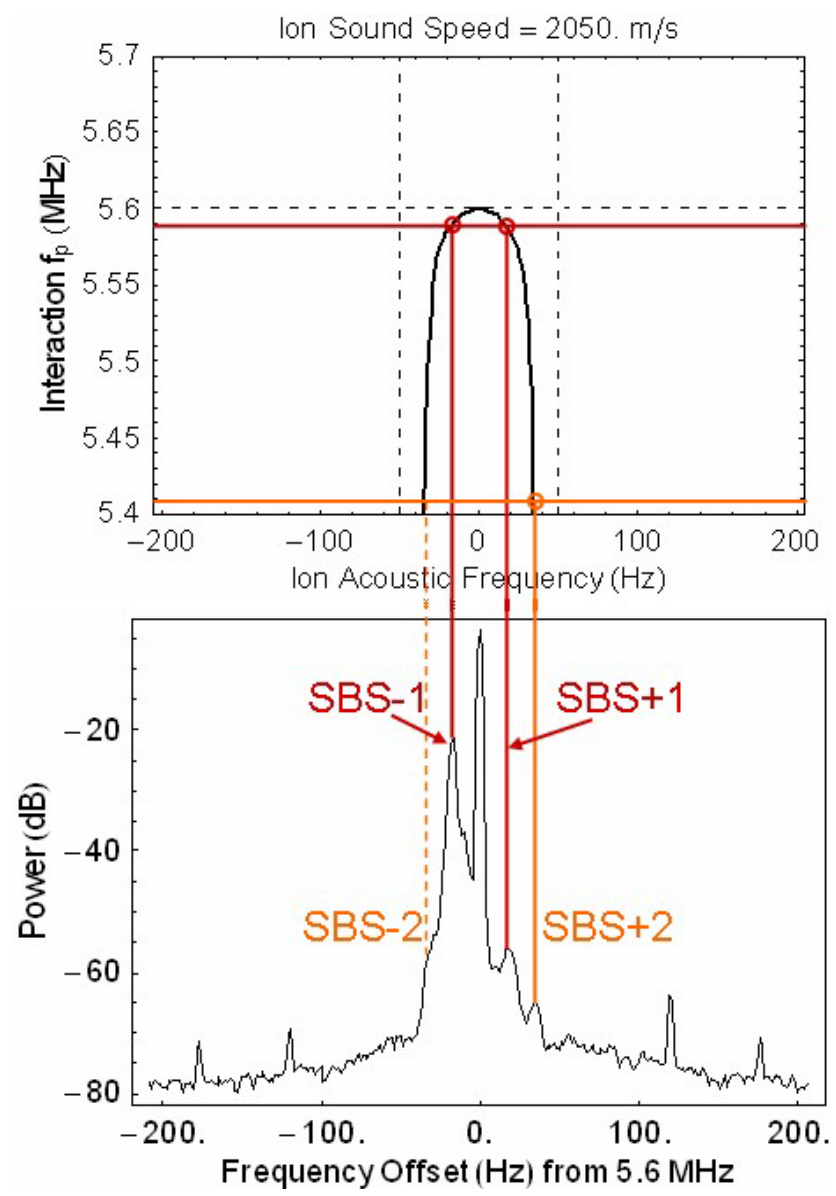

Fig. 14. Transfer of the low-frequency emission peaks from stimulated Brillouin scatter to the plasma frequencies in the ionospheric interaction regions. The top matching curve is computed assuming the ion-sound speed has a constant value of $2050 \mathrm{~m} / \mathrm{s}$ throughout the interaction region in the plasma.

tilt of the ray path. The results of this computation were compared to the measured SBS spectrum yielding an estimate of the plasma frequency at the interaction regions. The mapping between the ion-acoustic frequency offsets of the observed SBS spectra and the plasma frequency required for matching is illustrated in Fig. 14. The ion-sound speed $(2050 \mathrm{~m} / \mathrm{s})$ was adjusted to match the measurements for the SBS+2 peak with the theoretical transfer-function at the upper-hybrid altitude. The inflection in the downshifted side of the spectra near $-30 \mathrm{~Hz}$ maps an SBS-2 region that is masked by the strong SBS-1 emission.

Because of the small tilt angle for the HF beam and presence of the independence of the wave normal at the UH resonance level, the SBS matching theory applied to vertical incidence can also be applied to magnetic zenith excitations. The procedure for mapping the SEE spectra to the interaction altitude is demonstrated with the SBS matching curve in Fig. 14. Since no lines above the ion cyclotron frequency 


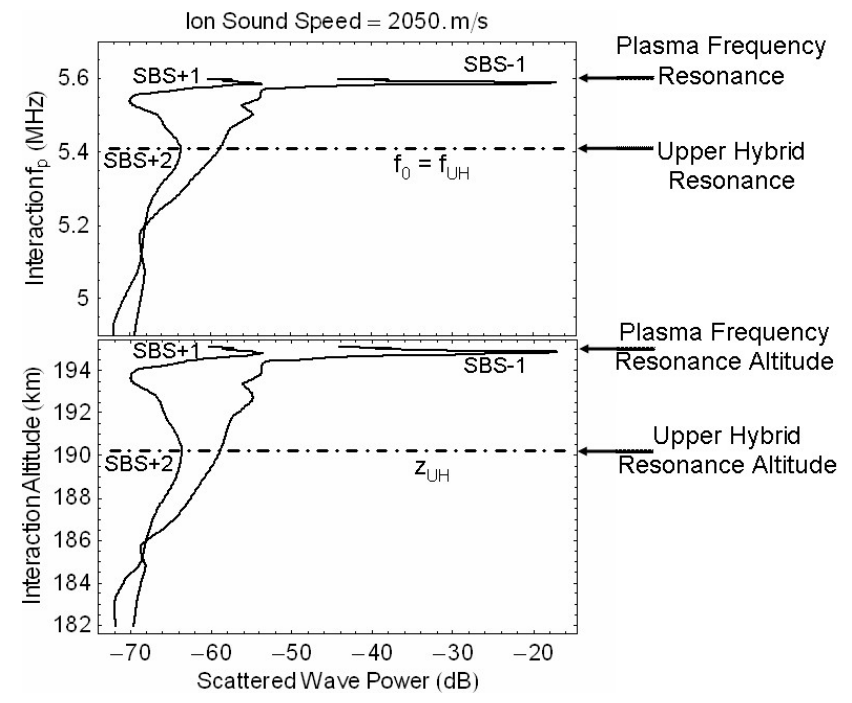

Fig. 15. Electromagnetic emission lines from the SBS spectrum mapped to the source altitudes for a magnetic zenith pump wave at 5.6 MHz. The two separate lines come from two distinct altitude regions. The peak strength is just below the reflection level and the dot-dash line is at the upper hybrid resonance altitude. The altitude determination is obtained from an assumed electron density profile.

are observed, the EIC portion of the matching curve is not shown. The SBS-1 and SBS+1 lines are much stronger than the corresponding lines (SBS-2 and SBS+2) from the UH level because the pump amplitude is much weaker at the $\mathrm{UH}$ level than near the reflection level where the plasma frequency is nearly equal to the $5.6 \mathrm{MHz}$ pump frequency.

Each feature of the SBS frequencies is transferred to the profile of the backscattered EM emission source region (Fig. 15). The maximum intensity of the SBS lines maps well to the altitude profiles of Sect. 5 where the strong maximum EM wave is expected just below the reflection level. The much weaker scatter EM wave is expected at the UH level where the pump is much weaker. The UH resonance interactions occur when the ion sound speed is set to $2050 \mathrm{~m} / \mathrm{s}$. This corresponds to an electron temperature of $4000 \mathrm{~K}$ using the assumptions for Eq. (27) along with the assumption that the elevated electron temperature is constant along a magnetic field line. With these measurements, it seems that the electron temperature at $19: 38$ using $5.6 \mathrm{MHz}$ with $1.66 \mathrm{GW}$ ERP produced a substantially larger electron temperature $(\sim 4000 \mathrm{~K})$ than was observed 10 and 20 min later with $4.5 \mathrm{MHz}$ at $1.0 \mathrm{GW} \mathrm{ERP}(\sim 3000$ to $3500 \mathrm{~K})$.

\section{Summary and conclusions}

This paper has provided experimental measurements of stimulated Brillouin scatter supported by wave matching theory and ray tracing. The measurements of stimulated Brillouin scattering have been made using a digital sampling re- ceiver located near the HAARP high power HF transmitter in Alaska. The emissions seem to originate either from the upper hybrid level or from the pump-maximum altitude just below the O-mode reflection or plasma-resonance level. The SBS data taken over a $20 \mathrm{~min}$ period from 19:40 to 20:00 UT (11:20 to 12:00 Local Time in Alaska) only show both downshifted (SBS-) and upshifted (SBS+) lines from near UH resonance region with $30 \mathrm{~Hz}$ offsets from the pump wave. In all cases with a $4.5 \mathrm{MHz}$ pump, the upshifted, anti-Stokes line is between 4 and $8 \mathrm{~dB}$ weaker than the downshifted, Stokes line. The positions of the lines do not show significant change either when the power of the pump is dropped for the vertical beam or when the full power pump is tilted toward the magnetic zenith.

The SBS data at $5.6 \mathrm{MHz}$ show strong interactions near the reflection level for magnetic zenith heating. This effect lasted for $20 \mathrm{~min}$ between 19:20 and 19:40 UT. The Stokes line was $37 \mathrm{~dB}$ larger than the anti-Stokes emission. A weak upshifted SBS+2 line in the spectra is located at an ion-acoustic frequency that is consistent with SBS matching conditions at the UH resonance altitude.

All the spectra also show a low frequency continuum extending $\pm 200 \mathrm{~Hz}$ from the pump frequency. The intensity of this low-frequency continuum is also larger on the downshifted side than the upshifted side of the pump wave. The low-frequency continuum cannot be produced by the SBS generation of either ion acoustic or electrostatic-ion cyclotron waves because the matching conditions do not permit frequencies above $f_{M+}=61 \mathrm{~Hz}$. The source of this continuum could be low-level spurious noise from the HAARP transmitter.

The theory for stimulated Brillouin scattering has been applied to regions of the ionosphere where the electromagnetic pump frequency exceeds the local value of plasma frequency. During SBS, the pump wave decays into a backscattered EM photon and a low-frequency phonon. Energy and momentum conservation as represented by the wave-wave interactions define the properties of the scattered electromagnetic wave. The conservation laws (Eq. 3) and dispersion equations for the low-frequency electrostatic wave (Eq. 6) and the scattered electromagnetic wave (Eq. 13) are solved to give the relationship between the plasma frequency (or plasma density) where the SBS interaction is occurring and the low frequency offset of the scatter EM wave. The matching conditions are dependent on (1) the ion sound speed in the plasma, (2) the electron and ion gyro frequencies, (3) the propagation angle relative to the magnetic field, and the EM pump frequency. For the generalized SBS in a magnetized plasma, low-frequency electrostatic wave can be either an electrostatic ion cyclotron wave with a frequency just above the ion cyclotron frequency $\left(\Omega_{i}\right)$ or ion-acoustic wave with a frequency below $\Omega_{i}$. Only SBS associated with ion acoustic waves have been measured during ionospheric modification experiments using high power HF waves. 
Useful expressions that relate the measured ion acoustic frequency offsets to the ion sound speeds and the electron temperatures at the interaction region is given by Eqs. (26) and (27), respectively. From this later expression, the electron temperature in the modified F-region plasma can be estimated using the SBS ion line offset frequency along with complementary data from the ionosonde to locate the altitude of the interaction regions. Also needed are magnetic field models to provide the electron and ion gyro frequencies as well as the propagation angle between the HF wave and the magnetic field direction. Electron temperature measurements in the ionosphere are usually provided by incoherent scatter radars (Djuth et al., 1987; Gustavsson et al., 2005). The Modular UHF Ionospheric Radar (MUIR) facility at HAARP can detect the enhanced scatter from artificial ion acoustic and electron plasma waves but it currently cannot be used as incoherent scatter radar (ISR) to provide direct measurements of electron temperatures. The SBS line technique provides a much needed alternative for electron temperature measurements. Future experiments at a high power HF facility with an ISR should attempt to provide validation of the electron temperature measurements in the HF interaction region using both the SBS and ISR techniques. Temporal evolution of the electron temperature immediately after pump turn-on should be detectable by shifts in the ionacoustic lines obtained by the SBS technique.

Using the theory for SBS matching conditions, it was shown that the measured scattered 4.5 MHz SBS EM waves map to the altitudes where upper hybrid frequency matches the pump frequency not near the altitude where the pump reflects. If the SBS occurred near the reflection altitude, the SBS spectrum would have lines less than $17 \mathrm{~Hz}$ from the pump frequency. When the SBS occurs at the UH level, the SBS spectra show lines around $30 \mathrm{~Hz}$ from the pump frequency. The stimulated Brillouin scatter experiments reported by Norin et al. (2009) show two distinct types of SBS spectra. When the HF beam was vertical, the wide spacing of the SBS lines with offset frequencies around $30 \mathrm{~Hz}$ was produced. When the HF beam was pointed to the magnetic zenith, very strong SBS lines were produced with lower frequency offset frequencies in the range of 9 to $15 \mathrm{~Hz}$ as well as wider spaced emissions with offset frequencies near $25 \mathrm{~Hz}$. Norin et al. (2009) attributed this to the change in the propagation from just below the reflection level to the height of the upper hybrid resonance. The theory in Sect. 4 agrees with this conclusion and has allowed interpretation of the frequency offsets to provide a measure of the electron temperature in the HF interaction region. The vertical heating results and the wide SBS frequency spacing presented by Norin et al. (2009) and both the vertical and magnetic-zenith results presented here came from interactions of the pump at the upper hybrid resonance level. The magnetic zenith results with narrow SBS frequencies are attributed to interactions just below the reflection altitude where large longitudinal electric fields are produced. The UH level interactions can produce the emissions called SBS-2 in this paper and 2np by Norin et al. (2009). Both works conclude that the second-harmonic cascade process illustrated by the left most panel in Fig. 8 is not responsible for the SBS-2 emission.

The matching condition theory has not considered the SBS growth rate. The threshold for SBS in an unmagnetized plasma is well known (Kruer, 1988; Eliezer, 2002) but the derivation should be repeated with the added complications of a magnetized plasma with the effects of electron and ion cyclotron resonances. This is another subject for a future paper.

The parametric instability excited by scattering off electrostatic ion cyclotron waves has been previously described by Shukla and Tagare (1979). The SBS matching conditions support the conclusion that the O-Mode wave may excite electrostatic ion cyclotron (EIC) waves slightly above the ion cyclotron frequency around $50 \mathrm{~Hz}$. The evidence of this emission is not seen in the current SEE observations. A search for this excitation will be part for future experiments at HAARP. Theoretic work on the SBS growth rate should compare the growth of the ion-acoustic mode with the growth of the EIC mode.

The theory has not explained the significance of the relative amplitudes of the anti-Stokes line relative to the Stokes line. A consistent model of the Stokes and anti-Stokes electromagnetic emissions should provide estimates of the strength of the ion-acoustic waves in the ionosphere. A future paper will explore this with a full simulation of SBS-line generation.

The discovery of stimulated Brillouin scatter in inhomogeneous magnetized plasmas provides both a new diagnostic tool for determining the state of the ionosphere modified by high power radio waves and an exciting new regime for investigation of nonlinear interactions of electromagnetic waves with the ionosphere. Such new discoveries are likely to continue with the new effective radiated powers of the HAARP transmitter and new ground diagnostics such as the GBOX5 receiver.

Acknowledgements. This research was funded at NRL by the Office of Naval Research Laboratory. The research was also supported in part by the High Frequency Aurora Research Program (HAARP) through the Air Force Research Laboratory (AFRL). The authors thank Steven P. Obenchain for discussions about laser plasma interactions.

Topical Editor M. Pinnock thanks T. Leyser and another anonymous referee for their help in evaluating this paper.

\section{References}

Budden, K. G.: Radio Waves in the Ionosphere, Cambridge University Press, New York, 1966.

Budden, K. G.: The Propagation of Radio Waves, Cambridge University Press, Cambridge, 1985. 
Djuth, F. T., Thide, B., Ierkic, H. M., and Sulzer, M. P.: Large F-region electron-temperature enhancements generated by highpower HF radio waves, Geophys. Res. Lett., 14, 953-956, 1987.

Eliezer, S.: The Interaction of High-Power Lasers with Plasmas, Institute of Physics, Bristol, UK, 2002.

Fejer, J. A.: Ionospheric modification and parametric instabilities, Rev. Geophys. Space Phys., 17, 135-153, 1979.

Försterling, K.: Über die Ausbrietung electromagnetischer Wallen in einem magnetisierten Medium bei senkrechter Inzidenz, Hochfrequenztech. Elekroakust, 59, 10-22, 1942.

Goldston, R. J. and Rutherford, P. H.: Introduction to Plasma Physics, CRC Press, 1995.

Gustavsson, B., Sergienko, T., Kosch, M. J., Rietveld, M. T., Brändström, B. U. E., Leyser, T. B., Isham, B., Gallop, P., Aso, T., Ejiri, M., Grydeland, T., Steen, Å., LaHoz, C., Kaila, K., Jussila, J., and Holma, H.: The electron energy distribution during HF pumping, a picture painted with all colors, Ann. Geophys., 23, 1747-1754, 2005, http://www.ann-geophys.net/23/1747/2005/.

Hazelgrove, J.: Ray Theory and a New Method of Ray Tracing, in: The Physics of the Ionosphere: Report of the Physical Society Conference on Physics of the Ionosphere, held at Cavendish Laboratory, Cambridge, September 1954, London, Physical Society, pp. 355-364, 1955.

Kruer, W. L.: The Physics of Laser Plasma Interactions, AddisonWesley, New York, 1988.

Leyser, T. B.: Stimulated Electromagnetic Emissions By HighFrequency Electromagnetic Pumping Of The Ionospheric Plasma, Space Sci. Rev., 98, 223-328, 2001.
Leyser, T. B., Norin, L., McCarrick, M., Pedersen, T. R., and Gustavsson, B.: Radio Pumping of Ionospheric Plasma with Orbital Angular Momentum, Phys. Rev. Lett., 102, 065004, doi:PhysRevLett 102.06504, 2009.

Lundborg, B. and Thide, B.: Standing wave pattern of HF radio waves in the ionospheric reflection region, 1. General Formulas, Radio Sci., 20, 947-958, 1985.

Lundborg, B. and Thide, B.: Standing wave pattern of HF radio waves in the ionospheric reflection region, 2. Applications, Radio Sci., 21, 486-500, 1986.

Mendonça, J. T., Thidé, B., and Then, H.: Stimulated Raman and Brillouin Backscattering of Collimated Beams Carrying Orbital Angular Momentum, Phys. Rev. Lett., 102, 185005, doi:PhysRevLett 102.185005, 2009.

Norin, L., Leyser, T. B., Nordblad, E., Thidé, B., and McCarrick, M.: Unprecedentedly strong and narrow electromagnetic emissions stimulated by high-frequency radio waves in the ionosphere, Phys. Rev. Lett., 102, 065003, doi:PhysRevLett 102.065003, 2009.

Ruck, G. T., Barrick, D. E., Stuart, W. D., and Krichbaum, C. K.: Radar Cross Section Handbook, Plenum Press, New York, 1970.

Shukla, P. K. and Tagare, S. G.: Scattering of ordinary electromagnetic waves by ion cyclotron waves in the ionosphere, J. Geophys. Res., 84, 1317-1318, 1979.

Stix, T. H.: Waves in Plasmas, AIP Press, 1992.

Yeh, K. C.: and CH Liu, Ionospheric Waves, Academic Press, 1972. 